\title{
Genome-wide characterization and phylogenetic analysis of GSK gene family in three species of cotton: evidence for a role of some GSKs in fiber development and responses to stress
}

Lingling Wang ${ }^{1,2}$, Zhaoen Yang ${ }^{1}$, Bin Zhang ${ }^{1}$, Daoqian Yu ${ }^{1}$ Ji Liu', Qian Gong ${ }^{1}$, Ghulam Qanmber ${ }^{1}$, Yi Li ${ }^{1}$, Lili Lu', Yongjun Lin' ${ }^{2}$ Zuoren Yang ${ }^{1 *}$ and Fuguang $\mathrm{Li}^{\mathrm{i}^{*}}$

\begin{abstract}
Background: The glycogen synthase kinase 3/shaggy kinase (GSK3) is a serine/threonine kinase with important roles in animals. Although GSK3 genes have been studied for more than 30 years, plant GSK genes have been studied only since the last decade. Previous research has confirmed that plant GSK genes are involved in diverse processes, including floral development, brassinosteroid signaling, and responses to abiotic stresses.

Result: In this study, 20, 15 (including 5 different transcripts) and 10 GSK genes were identified in G. hirsutum, G. raimondii and G. arboreum, respectively. A total of 65 genes from Arabidopsis, rice, and cotton were classified into 4 clades. High similarities were found in GSK3 protein sequences, conserved motifs, and gene structures, as well as good concordance in gene pairwise comparisons (G. hirsutum vs. G. arboreum, G. hirsutum vs. G. raimondii, and G. arboreum vs. G. raimondii) were observed. Whole genome duplication (WGD) within At and Dt sub-genomes has been central to the expansion of the GSK gene family. Furthermore, GhSK genes showed diverse expression patterns in various tissues. Additionally, the expression profiles of GhSKs under different stress treatments demonstrated that many are stress-responsive genes. However, none were induced by brassinolide treatment. Finally, nine co-expression subnetworks were observed for GhSKs and the functional annotations of these genes suggested that some GhSKs might be involved in cotton fiber development.

Conclusion: In this present work, we identified 45 GSK genes from three cotton species, which were divided into four clades. The gene features, muti-alignment, conversed motifs, and syntenic blocks indicate that they have been highly conserved during evolution. Whole genome duplication was determined to be the dominant factor for GSK gene family expansion. The analysis of co-expressed sub-networks and tissue-specific expression profiles suggested functions of GhSKs during fiber development. Moreover, their different responses to various abiotic stresses indicated great functional diversity amongst the GhSKs. Briefly, data presented herein may serve as the basis for future functional studies of GhSKs.
\end{abstract}

Keywords: GSK3 (glycogen synthase kinase 3/shaggy kinase), Cotton, Genome duplication, Fiber development, Abiotic stress, Co-expression network

\footnotetext{
* Correspondence: yangzuoren4012@163.com; aylifug@163.com

${ }^{1}$ State Key Laboratory of Cotton Biology, Key Laboratory of Biological and

Genetic Breeding of Cotton, Institute of Cotton Research, Chinese Academy

of Agricultural Sciences, Anyang 455000, Henan, China

Full list of author information is available at the end of the article
}

(c) The Author(s). 2018 Open Access This article is distributed under the terms of the Creative Commons Attribution 4.0 International License (http://creativecommons.org/licenses/by/4.0/), which permits unrestricted use, distribution, and reproduction in any medium, provided you give appropriate credit to the original author(s) and the source, provide a link to the Creative Commons license, and indicate if changes were made. The Creative Commons Public Domain Dedication waiver (http://creativecommons.org/publicdomain/zero/1.0/) applies to the data made available in this article, unless otherwise stated. 


\section{Background}

Glycogen synthase kinase 3 (GSK3), which is also known as the SHAGGY-like protein kinase, is a non-receptor serine/threonine protein kinase and involved in vital signal transduction pathways in eukaryotes $[1,2]$. In mammals, GSK3 exists as two isoforms, namely GSK3 $\alpha$ and GSK3 $\beta$, both of which help to regulate glycogen metabolism [3]. GSK3 was first reported as an enzyme-inactivating kinase in rabbit skeletal muscle [4]. The products of GSK3 homologs were confirmed to be involved in several physiological processes in animals, including protein synthesis, glycogen metabolism, regulation of transcription factor activity, determination of cell fate, and tumorigenesis [5-8]. Additionally, GSK3 is a key component of the animal Wnt signaling pathway $[9,10]$.

The plant GSK3 genes appear to be more diverse than the corresponding animal genes, as evidenced by the fact that there are ten Arabidopsis thaliana genes and nine rice genes [1]. The plant GSK3 genes were as diverse in function as animal isoforms. Biochemical and genetic analyses demonstrated that different plant GSK3 members affect disparate processes, such as development, stress responses, and signaling pathways, by phosphorylation of different protein substrates. In A. thaliana, AtSK11 and AtSK12 were highly expressed during the early stages of differentiation of the floral primordium. These two genes were subsequently expressed in specific regions, including the pollen-containing area of the anther, carpels, petals, and sepal primordial cells [11]. Previous studies revealed that AtSK31, which was localized mainly to the nuclei of developing tissues, was highly expressed in floral organs [12, 13]. Moreover, overexpression of different AtSK32 isoforms in A. thaliana resulted in different phenotypes. Two mutations of AtSK32, Lys167, and Arg178 (homologous to the critical active site residues Lys85 and Arg96 of mammal GSK3 3 ) were generated by site-directed mutagenesis [14]. However, overexpression of wild-type or catalytically inactive mutant (i.e., encoding a K167A mutation) AtSK32 gene in $A$. thaliana produced no observable effects. Nevertheless, overexpression of AtSK32-R178A displayed shorter pedicels and smaller petals compared with wild-type controls [14]. Moreover, ASK $\alpha /$ AtSK11, which was an $A$. thaliana GSK3, regulated stress resistance by activating Glc-6-phosphate dehydrogenase (G6PD). Increased G6PD activity accompanied with decreased reactive oxygen species levels have been observed in $A S K \alpha$-overexpressing plants under stress conditions, especially during salt stress [15]. GSK3/SHAGGY-like kinase (AtSK21) gain-of-function mutations or over-expressing transgenetic lines inhibited the brassinosteroid (BR) signaling pathway and led to BR-deficiency and suppression of BR-induced responses [16]. Upregulating the expression level of OsGSK3 in rice (Oryza sativa L. 'Nipponbare') enhanced its tolerance to salt, cold, drought and mechanical injury, and abscisic acid (ABA) responsiveness [6]. There are ongoing studies aimed at comprehensively examining GSK3 family members in various plant species, even though the number of putative GSK3 genes is relatively low.

Cotton fibers, which are the premier natural fiber derived from the seedcoat epidermal cells, are the most important renewable resource used by the textile industry. The genus Gossypium that includes approximately 50 species, of which four are cultivated for their cotton fibers (i.e., G. arboreum and G. herbaceum, $2 \mathrm{n}=2 \mathrm{X}=\mathrm{AA}=26$; $\mathrm{G}$. hirsutum (upland cotton), and G. barbadense (sea island cotton), $2 \mathrm{n}=4 \mathrm{X}=\mathrm{AADD}=52$ ) [17]. Gossypium raimondii, another diploid, carries a D-genome and only produces very short and coarse seed fibers [17]. Gossypium hirsutum is the most widely cultivated cotton species and accounts for more than $90 \%$ of the global cotton fiber yield [18]. This allotetraploid species contains two different sets of chromosomes (i.e., A and D) evolved as a result of inter-specific hybridization during the Pleistocene about 1-2 million years ago $[17,19]$. Cotton fiber development is a complicated process involving several phytohormones, including auxin, ethylene, gibberellins (GAs), and BRs [20-25]. The BIN2 protein, as a negative regulator of BR signaling pathway, was a well-characterized GSK3 that could affect the activities of phytohormone-signaling pathways [26-32]. Additionally, the complete genome sequencing of G. hirsutum, G. arboreum and G. raimondii now allow genome-wide analyses of any gene family in cotton [19, 33-36]. However, there have been no systematic investigations of the GSK gene family from these three cotton species. Considering the functional importance of the GSK gene family, we conducted an in silico genome-wide search and analysis to identify and characterize the GSK gene family members of G. arboreum, G. hirsutum, and G. raimondii. We subsequently analyzed a multi-sequence alignment, gene loci, gene structures, promoter cis-elements, conserved protein motifs, phylogenetic relationships, gene expression profiles, and a weighted gene co-expression sub-network analysis. The results described herein may be useful for further functional characterizations of cotton GSKs. Our data may also help to clarify the mechanism underlying the regulatory effects of GSKs during cotton development, growth, and responses to stress conditions.

\section{Methods}

\section{Plant materials and growth conditions}

Gossypium hirsutum L. 'cv CCRI24' plants were grown in mixed soil under glasshouse conditions [14 h light $\left(28 \sim 34{ }^{\circ} \mathrm{C}\right) / 10 \mathrm{~h}$ dark $\left.\left(24 \sim 27^{\circ} \mathrm{C}\right) ; 150 \mu \mathrm{mol} \mathrm{m}^{-2} \mathrm{~s}^{-1}\right]$. To analyze organ- and tissue-specific gene expression patterns in 'CCRI24', plants were grown under field conditions in Zhengzhou, China following standard crop 
management practices. Flower (whole flower at $0 \mathrm{dpa}$ ) and ovule samples were collected at 1, 3, 5 days post-anthesis (dpa). Additionally, isolated fibers were collected at 7, 10, 15, and $20 \mathrm{dpa}$. Stages of the tissues collected were chosen as described in previously published research [34, 37]. Three biological replicates were collected for each sample. All collected samples were immediately frozen in the liquid nitrogen and stored at $-80^{\circ} \mathrm{C}$ for RNA extraction and subsequent analysis.

\section{Abiotic stress assays and BL treatment}

The expression patterns of GhSK genes in response to various environmental stresses and brassinolide treatment were analyzed. For abiotic stresses, phenotypically similar G. hirsutum potted seedlings grown in a glasshouse up to the three-true-leaves stage (four weeks old) were selected [38, 39]. Each treatment consisted of three biological replicates of the individual seedling. For brassinolide treatment, seedlings were cultured in deionized water supplemented with $10 \mu \mathrm{M}$ brassinolide, and leaf samples were collected at $0,0.5,1,3,5 \mathrm{~h}$. The cold and heat treatments involved incubations at 4 and $38{ }^{\circ} \mathrm{C}$, respectively. To simulate dehydration stress, cotton seedlings were irrigated with $20 \%$ polyethylene glycol (PEG) instead of water. Additionally, cotton seedling roots were immersed in $300 \mathrm{mM} \mathrm{NaCl}$ solutions to assess the effects of salt stress. The true leaves were collected at $1,3,6$, and $12 \mathrm{~h}$ for all of the abiotic stresses performed as described in previously published investigations [38, 39]. All collected leaf samples were immediately frozen in liquid nitrogen and stored at $-80^{\circ}$ $\mathrm{C}$ for subsequent RNA isolation and cDNA synthesis.

\section{RNA isolation and quantitative real-time polymerase chain reaction analysis}

Total RNA was extracted from collected cotton tissues/ organs using the RNAprep Pure Plant Kit (TIANGEN, Beijing, China). First-strand cDNA was synthesized using the PrimeScript ${ }^{\text {tw }}$ RT Reagent Kit, during which the genomic DNA was eliminated with gDNA Eraser (Perfect Real Time; Takara, Dalian, China). The quantitative real-time polymerase chain reaction (qRT-PCR) primers were designed by the Primer Premier 5.0 software. Histone 3 (GenBank accession no. AF024716) was selected as the reference gene. The qRT-PCR was conducted using SYBR Premix Ex Taq ${ }^{\text {ma }}$ (Tli RNase H Plus) (Takara) and ABI 7900 qRT-PCR System (Applied Biosystems, CA, USA). The PCR program was as follows: $95^{\circ} \mathrm{C}$ for $30 \mathrm{~s} ; 40$ cycles of $95^{\circ} \mathrm{C}$ for $5 \mathrm{~s}$ and $60^{\circ} \mathrm{C}$ for 20 $\mathrm{s}$. The $2^{-\Delta \Delta \mathrm{Ct}}$ method was applied to calculate the relative expression level of all target genes as compared to control treatments [40].

\section{Identification of cotton GSK genes}

The latest version of the A. thaliana and rice genome, protein sequence and annotation databases were downloaded at http://www.arabidopsis.org/ and http://plants.ensembl.org/index.html [41], respectively. Additionally, the genome sequence versions of the G. hirsutum (NAU, Version 1.1) [34], G. raimondii (JGI, version2.0) [19] available from COTTONGEN (https://www.cottongen.org) [42] and G. arboreum (PacBio-Gar-Assembly-v1.0, $\mathrm{ftp}$ ://bioinfo.ayit.edu.cn/downloads/) [43] were used to identify GSK proteins and their corresponding nucleotide sequences. The cotton GSK genes and encoded proteins were identified via a BLAST search using all of the A. thaliana ASK gene and encoded protein sequences as queries. For protein analyses, the following parameters were used: e-value $=1 \mathrm{e}-5$ and coverage ratio $=50 \%$. Moreover, the definition of the Pkinase (PF00069.23) domain was downloaded from Pfam: http://pfam.janelia.org/ [44], and then the hidden Markov model (HMM) was used to verify the GSKs from the three cotton species. The identified A. thaliana, rice, G. hirsutum, G. arboreum, and G. raimondii genes were renamed as previously reported [45] based on their order of phylogenetic clustering.

\section{Multiple-sequence alignment and phylogenetic analysis}

A sequence alignment of full-length protein sequences from the three analyzed cotton species, A. thaliana, and rice was prepared using MUSCLE: http://www.ebi.ac.uk/Tools/msa/muscle/ and saved in the ClustalW format. Meanwhile, a phylogenetic analysis of the A. thaliana, rice, and cotton GSKs was conducted using the MEGA 6.0 program, with 1000 bootstrap replications [46]. An unrooted Neighbor-joining, as well as Minimum-Evolution tree were constructed using the Poisson model method using the same alignment file.

\section{Comparison of chromosomal distributions, exon/ intron structures, and protein domains among a, D, and AD cotton genomes}

The genomic distribution of the cotton GSK genes was analyzed by MapInspect software (https://mapinspect1.software.informer.com/) according to the start positions indicated in G. hirsutum, G. arboreum, and G.raimondii databases [47]. The intron/ exon structures were examined using the Gene Structure Display Server 2.0 program (http:/gsds.cbi.pku.edu.cn/). Meanwhile, the cotton GSK domains were predicted with the MEME (Multiple Expectation Maximization for Motif Elicitation) online tool: http://meme-suite.org/tools/meme [48] using the following parameters: motif width $6-200$ residues and the maximum number of motifs $=20$. The mast.xml file exported from MEME was used to generate the motif images using 
TBtools_master (version 0.49991) (https://github.com/ CJ-Chen/TBtools). The conserved motif logos were downloaded from MEME as well.

\section{Synteny and gene duplications analysis}

The G. hirsutum, G. arboreum, and G. raimondii genome data were searched using a BLAST-Like Alignment Tool (BLAT) [49] to identify tandem duplications which were defined as multilocus genes located in adjacent regions or separated by uniform intergenic regions. Sequences with coverage $>90 \%$ and similarity $>95 \%$ were designated as tandem duplicates.

We performed the synteny analysis of GSK genes among the three cotton species included in this study (i.e., G. hirsutum vs. G. arboreum, G. hirsutum vs. G. raimondii, and $G$. arboreum vs. G. raimondii). BLAT was used for the pairwise comparison of G. hirsutum, G. arboreum, and G. raimondii gene sets and to identify the homologous genomic regions. Finally, the syntenic blocks were visualized using the default parameters of the Circos (version 0.69) program [50].

\section{Analysis of the promoter cis-regulatory elements}

The 2-kb sequences upstream of the cotton GhSK genes (i.e., putative promoter regions) were obtained by BLAST searches of the cotton genome data using whole gene IDs. Potential cis-acting regulatory elements of the extracted sequences were subsequently subjected to PlantCARE database analysis (http://bioinformatics.psb.ugent.be/webtools/plantcare/html/) [51]. The identified cis-elements were drawn using a custom script in the $\mathrm{R}$ program (version 3.20; https://www.r-project.org/).

\section{Biophysical properties of GSK genes from three cotton species}

The three genome sets of cotton GSK protein sequences were analyzed using ExPASy-ProtParam tool: http://web.expasy.org/protparam/ to calculate the number of amino acids, molecular weight, and theoretical pI [52]. Meanwhile, the subcellular localization of these genes was predicted with ProComp 9.0 (http://www.softberry.com/berry.phtml?group $=$ programs\&subgroup $=$ proloc\&topic $=$ protcomppl) [53].

\section{Weighted co-expression sub-network analysis of GhSK genes}

The weighted gene co-expression sub-network analysis (WGCNA) of the GhSK genes was conducted using the G. hirsutum TM-1 transcriptome sequencing data downloaded from the NCBI Sequence Read Archive database $[34,54]$. The network construction and module detection were performed using the 'cuttreeDynamic' and 'mergeCloseModules' by "WGCNA" R package (version 1.4.9) [55], the parameters were set as follows: The power was 9; the minModuleSize was 30 and the cutHeight was 0.25. Finally, we visualized the sub-network using Cytoscape (version 3.4.0) program [56]. An additional investigation of the putative functions of GhSK genes and their co-expression genes was based on Gene Ontology (GO) and Kyoto Encyclopedia of Genes and Genomes (KEGG) enrichment analyses.

\section{Gene expression patterns analyzed using published transcriptomic data}

The high-throughput G. hirsutum TM-1 transcriptome sequencing data were used to investigate the GhSK gene expression patterns in vegetative tissues, fiber tissues, floral organs, and dry seed. The log10 transformed Fragments Per Kilobase of transcript per Million fragments (FPKM) mapped values of the 20 GhSK genes were calculated to generate heatmaps with the local Multiple Arrays Viewer program (http://www.mybiosoftware.com/mev-4-6-2-multiple-experiment-viewer.html). The accession numbers and related sample information of the data used in this study are listed in Additional file 1: Table S1.1, S1.2 and Additional file 2: Table S2.

\section{Statistical analysis}

Data analyses here were executed through one-way analysis of variance (ANOVA) and a Dunnett's test at $p<0.05$ was performed. Taking the biological significance of the differential expression into account, we assumed a two-fold cut-off value for analyzing the stress and hormone induction or inhibition [57]. The expression levels were designated as 'induced' or 'inhibited' only when the differences met the specified criteria.

\section{Results}

Genome-wide characterization of cotton GSK genes

After removing redundant sequences, 20, 10, and 15 GSK genes were identified in G. hirsutum, G. arboreum, and G. ramondii genomes, respectively. To avoid the possibility of confusion and overlap with the gene names used, we renamed these genes as GhSKs, GaSKs, and GrSKs. The genes were numbered sequentially according to the subfamilies to which they were assigned after phylogenetic analysis (Table 1).

The biophysical characteristics of the identified cotton GSKs are provided in Table 1. The number of amino acids of putative GhSK, GaSK, and GrSK proteins varied from 376 (GhSK25 and GaSK22) to 494 (GhSK36), and the associated molecular weights ranged from 42.59 to $53.14 \mathrm{kDa}$ respectively. All pIs of the GSKs were higher than 7, except GhSK35 (6.81). Interestingly, the predicted subcellular localization revealed that almost all of the proteins were localized to multiple compartments including the cytoplasm, nucleus, and membrane, 
Table 1 Characteristics of the putative cotton GSK genes

\begin{tabular}{|c|c|c|c|c|c|c|c|}
\hline Subfamily & Gene ID & Proposed name & Amino acid length & $\mathrm{MW}(\mathrm{KDa})$ & $\mathrm{PI}$ & Subcellular location & Location \\
\hline \multirow[t]{9}{*}{ Clade I } & Gh_D11G2830 & GhSK11 & 407 & 46.20 & 8.59 & Cyto_Nucl_Memb & D24: 58071261-58,074,075(-) \\
\hline & Gh_A11G3270 & GhSK12 & 407 & 46.20 & 8.59 & Cyto_Nucl_Memb & Scaf3045_A11: 160892-163,705(-) \\
\hline & Gh_A12G1106 & GhSK13 & 409 & 46.41 & 8.69 & Cyto_Nucl_Memb & A12: 64114146-64,116,909(+) \\
\hline & Gh_D12G1230 & GhSK14 & 409 & 46.31 & 8.58 & Cyto_Nucl_Memb & D25: 40170076-40,172,846(+) \\
\hline & Ga11G0763 & GaSK11 & 407 & 46.23 & 8.59 & Cyto_Nucl_Memb & Ga1 1:13088074-13,090,884(+) \\
\hline & Ga12G1683 & GaSK12 & 409 & 46.37 & 8.58 & Cyto_Nucl_Memb & Ga12:26213850-26,216,616(+) \\
\hline & Gorai.007G308500.1 & GrSK11 & 407 & 46.20 & 8.59 & Cyto_Nucl_Memb & Gr07:52192786-52,197,103(-) \\
\hline & Gorai.007G308500.3 & GrSK11-1 & 407 & 46.20 & 8.59 & Cyto_Nucl_Memb & Gr07:52192786-52,197,103(-) \\
\hline & Gorai.008G136600.1 & GrSK12 & 409 & 46.36 & 8.58 & Cyto_Nucl_Memb & Gr08:38585110-38,589,107(+) \\
\hline \multirow[t]{14}{*}{ Clade II } & Gh_D06G2142 & GhSK21 & 381 & 43.24 & 8.74 & Cyto_Nucl_Memb & D19:63261021-63,263,915(-) \\
\hline & Gh_D09G2469 & GhSK22 & 382 & 43.10 & 8.74 & Cyto_Nucl_Memb & Scaf4332_D22:137949-141,233(-) \\
\hline & Gh_D09G2468 & GhSK23 & 381 & 43.38 & 8.83 & Cyto_Nucl_Memb & Scaf4332_D22:123124-126,295(-) \\
\hline & Gh_A09G0713 & GhSK24 & 381 & 43.32 & 9.02 & Cyto_Nucl_Memb & A09:52861382-52,864,569(+) \\
\hline & Gh_A09G0712 & GhSK25 & 376 & 42.64 & 8.88 & Cyto_Nucl_Memb & A09:52841598-52,844,894(+) \\
\hline & Gh_A06G2020 & GhSK26 & 385 & 43.72 & 8.69 & Cyto_Nucl_Memb & Scaf1340_A06:43052-45,877(+) \\
\hline & Ga06G2433 & GaSK21 & 381 & 43.24 & 8.74 & Cyto_Nucl_Memb & Ga06:130210718-130,213,627(+) \\
\hline & Ga09G0899 & GaSK22 & 376 & 42.59 & 8.84 & Cyto_Nucl_Memb & Ga09:59971361-59,974,663(+) \\
\hline & Ga09G0900 & GaSK23 & 382 & 43.55 & 8.89 & Cytoplasmic & Ga09:59988594-59,991,790(+) \\
\hline & Gorai.010G241600.3 & GrSK21 & 381 & 43.24 & 8.74 & Cyto_Nucl_Memb & Gr10:61087060-61,090,705(-) \\
\hline & Gorai.010G241600.2 & GrSK21-1 & 381 & 43.24 & 8.74 & Cyto_Nucl_Memb & Gr10:61085419-61,090,069(-) \\
\hline & Gorai.006G089300.1 & GrSK22 & 382 & 43.22 & 8.74 & Cyto_Nucl_Memb & Gr06:32530678-32,535,037(+) \\
\hline & Gorai.006G089300.2 & GrSK22-1 & 381 & 43.10 & 8.74 & Cyto_Nucl_Memb & Gr06:32530521-32,535,037(+) \\
\hline & Gorai.006G089400.1 & GrSK23 & 381 & 43.34 & 8.74 & Cyto_Nucl_Memb & Gr06:32546669-32,551,023(+) \\
\hline \multirow[t]{12}{*}{ Clade III } & Gh_A08G0285 & GhSK31 & 469 & 52.95 & 8.75 & Cyto_Nucl_Memb & A08:3329052-3,334,479(-) \\
\hline & Gh_D08G1440 & GhSK32 & 471 & 53.14 & 8.38 & Cyto_Nucl_Memb & D21:47361120-47,366,590(+) \\
\hline & Gh_D11G0907 & GhSK33 & 470 & 53.00 & 7.27 & Cyto_Nucl_Memb & D24:7839230-7,844,650(+) \\
\hline & Gh_A08G1158 & GhSK34 & 471 & 53.06 & 8.2 & Cyto_Nucl_Memb & A08:81021333-81,025,764(+) \\
\hline & Gh_A11G0778 & GhSK35 & 470 & 52.87 & 6.81 & Cyto_Nucl_Memb & A11:7649123-7,654,545(+) \\
\hline & Gh_D08G0378 & GhSK36 & 494 & 55.85 & 8.99 & Cyto_Nucl_Memb & D21:3871236-3,880,133(-) \\
\hline & Ga08G0389 & GaSK31 & 469 & 52.95 & 8.2 & Cyto_Nucl_Memb & Ga08:4054818-4,060,304(-) \\
\hline & Ga11G3164 & GaSk32 & 470 & 52.88 & 7 & Cyto_Nucl_Memb & Ga1 1:116299523-116,304,934(-) \\
\hline & Ga08G1552 & GaSK33 & 471 & 53.10 & 8.2 & Cyto_Nucl_Memb & Ga08:103960877-103,965,308(+) \\
\hline & Gorai.004G042400.1 & GrSK31 & 469 & 52.97 & 8.86 & Cyto_Nucl_Memb & Gr04:3659148-3,665,477(-) \\
\hline & Gorai.007G096100.1 & GrSK32 & 470 & 53.04 & 7.6 & Cyto_Nucl_Memb & Gr07:7076952-7,083,244(+) \\
\hline & Gorai.004G156700.2 & GrSK33 & 415 & 47.04 & 8.21 & Cyto_Nucl_Memb & Gr04:444455510-44,453,846(+) \\
\hline \multirow[t]{10}{*}{ Clade IV } & Gh_A01G1558 & GhSK41 & 422 & 47.85 & 8.63 & Cyto_Nucl_Memb & A01:92410497-92,413,873(+) \\
\hline & Gh_D01G1809 & GhSK42 & 422 & 47.85 & 8.63 & Cyto_Nucl_Memb & D14:55472543-55,475,936(+) \\
\hline & Gh_D12G0407 & GhSK43 & 422 & 47.90 & 8.49 & Cyto_Nucl_Memb & D25:6533937-6,537,332(-) \\
\hline & Gh_A12G0411 & GhSK44 & 422 & 47.87 & 8.49 & Cyto_Nucl_Memb & A12:8244999-8,248,430(-) \\
\hline & Ga02G1328 & GaSk41 & 422 & 47.84 & 8.63 & Cyto_Nucl_Memb & Ga02:91572951-91,576,357(+) \\
\hline & Ga12G2665 & GaSK42 & 439 & 50.02 & 8.68 & Cyto_Nucl_Memb & Ga12:98904726-98,909,799(-) \\
\hline & Gorai.002G218100.1 & GrSK41 & 422 & 47.85 & 8.63 & Cyto_Nucl_Memb & Gr02:56980865-56,985,951(+) \\
\hline & Gorai.008G045800.6 & GrSK42 & 422 & 47.89 & 8.49 & Cyto_Nucl_Memb & Gr08:6198704-6,202,924(-) \\
\hline & Gorai.008G045800.3 & GrSK42-1 & 422 & 47.89 & 8.49 & Cyto_Nucl_Memb & Gr08:6198649-6,203,828(-) \\
\hline & Gorai.008G045800.2 & GrSK42-2 & 422 & 47.89 & 8.49 & Cyto_Nucl_Memb & Gr08:6198649-6,203,828(-) \\
\hline
\end{tabular}


excepting for GaSK23 (only cytoplasmic). The localization results for the cotton GSKs were not the same as those that have been reported for Arabidopsis GSKs which might be because the cotton GSKs are much less well studied and their functions remain largely unknown.

\section{Phylogenetic analysis amongst the Arabidopsis thaliana, rice and cotton GSK genes}

To reveal the phylogenetic relationships among the GSK genes, full-length $A$. thaliana, rice, and cotton protein sequences were aligned. The aligned GSKs were highly similar and the conserved GSK protein kinase region is presented in detail in Additional file 3: Figure S1. Additionally, the kinase domain [2] and the tyrosine residue essential for kinase activity [2] were conserved among all the cotton GSKs.

A phylogenetic tree was generated using the neighbor-joining (NJ) (Figs. 1 and 3a) as well as minimum-evolution (ME) (Additional file 4: Figure S2) method of MEGA6.0. As shown in Figs. 1 and 3a, all sequences were divided into four subgroups (I, II, III, and IV), similar to previous studies [11, 12, 58, 59]. The phylogenetic tree indicated that GhSK, GaSK, and GrSK genes clustered together within each sub-group suggesting a close evolutionary affinity amongst the three cotton species and providing further support for the origins of the tetraploid species from a relatively recent hybridisation between an A-genome progenitor similar to $G$. arboreum and a D-genome progenitor similar to G. raimondii [33].

\section{Chromosomal locations, gene structures, and conserved motifs analysis}

Chromosomal locations of the cotton GSK genes using sequencing information for G. arboreum, G. raimondii, and G. hirsutum TM-1 revealed that the genes were unevenly distributed among chromosomes (Fig. 2). Out of the total of 45 cotton GSK genes, four GhSKs (GhSK12, GhSK23, GhSK24, and GhSK26) were assigned to scaffolds not connected to chromosomes (Fig. 2). The other 16 GhSK genes were distributed to chromosomes A08, A09 (two genes on each), A12 (two genes), D21 (two genes), D24, D25 (two genes on each), A01, A11, D14 and D19 (Fig. 2a). The GaSK genes were localized to chromosomes Ga02, Ga06, and Ga08, Ga11, Ga09 as well as Ga12 (two genes on each) (Fig. 2b), while GrSK genes were positioned on chromosomes Gr02 (one gene), Gr04 and Gr10 (two genes on each), Gr06 as well as Gr07 (three genes on each) and Gr08 (four genes) (Fig. 2c).

To further clarify the evolutionary relationships (Fig. 3a) among cotton GSK genes, genomic information corresponding to 40 (excluding the five different transcripts of GrSKs) cotton GSK genes extracted from Generic Feature Format (gff3) files were used to examine the structural diversity associated with the GhSK, GaSK, and GrSK genes. The structural analysis (Fig. 3b) indicated that the coding regions of all the cotton GSK genes were interrupted by 11-13 introns. The UTR regions of GhSK34, 35, and 36 and GaSK23 as well as GaSK33 are undetermined, while other cotton GSK genes had UTR region information in the genomic annotation files. In most cases, GSK genes within the same sub-family had similar gene structures in regard to the number and length of exons.

Using the MEME online server, 16 conserved domains and protein motifs were observed among 40 cotton GSKs (Fig. 3c), and the respective motif logos are presented in Additional file 5: Figure S3. Members of the same subfamily mostly contained the same motif components, suggesting they might have identical functions. Motifs 1-4 were found in all 40 analyzed GSKs, and constituted the kinase domain of GSK3. Additionally, motif 8 and 10 were present in all proteins of subfamily I and III. Motif 6 was observed in the members of subfamily III and IV. Furthermore, motif 13 was mainly present in subfamily II and IV members (except for GaSK22, GhSK25, and GrSK33), while motif 11 was identified in the proteins of subfamily I and IV. Motif 9 was mainly present in subfamily III members (except for GrSK33). Motif 14, 15, and 16 were present only in some members of subfamily III. The variations in the distribution of motifs indicate that cotton GSKs experienced functional diversification during their evolution.

\section{Duplicated cotton GSK genes and syntenic blocks}

During evolution, the two major mechanisms that generate novel genes and contribute to the complexity of the genomes of higher plants are small-scale tandem and large segmental duplications [60]. In this study, 25 pairs of paralogous GSK genes resulting from gene duplication events were identified in $G$. hirsutum (Table 2). In contrast, G. arboreum and G. raimondii consisted of 10 and 22 pairs of paralogs, respectively. Among the 25 pairs of duplicated GhSK genes, 14 were observed between different chromosomes, from which 8 pairs were identified between the At and Dt subgenomes. Further, 6 out of the 8 duplicated gene pairs were located between the homologous chromosomes of G. hirsutum [34]. GhSK24, GhSK25, and their paralogs were localized to chromosome A09. Other gene pairs were duplicated either between At to At, or Dt to Dt subgenomes, which might have resulted from the whole genome duplication (WGD) during plant genome evolution.

Syntenic blocks consist of conserved genes arranged similarly in chromosomes of different species. In this 


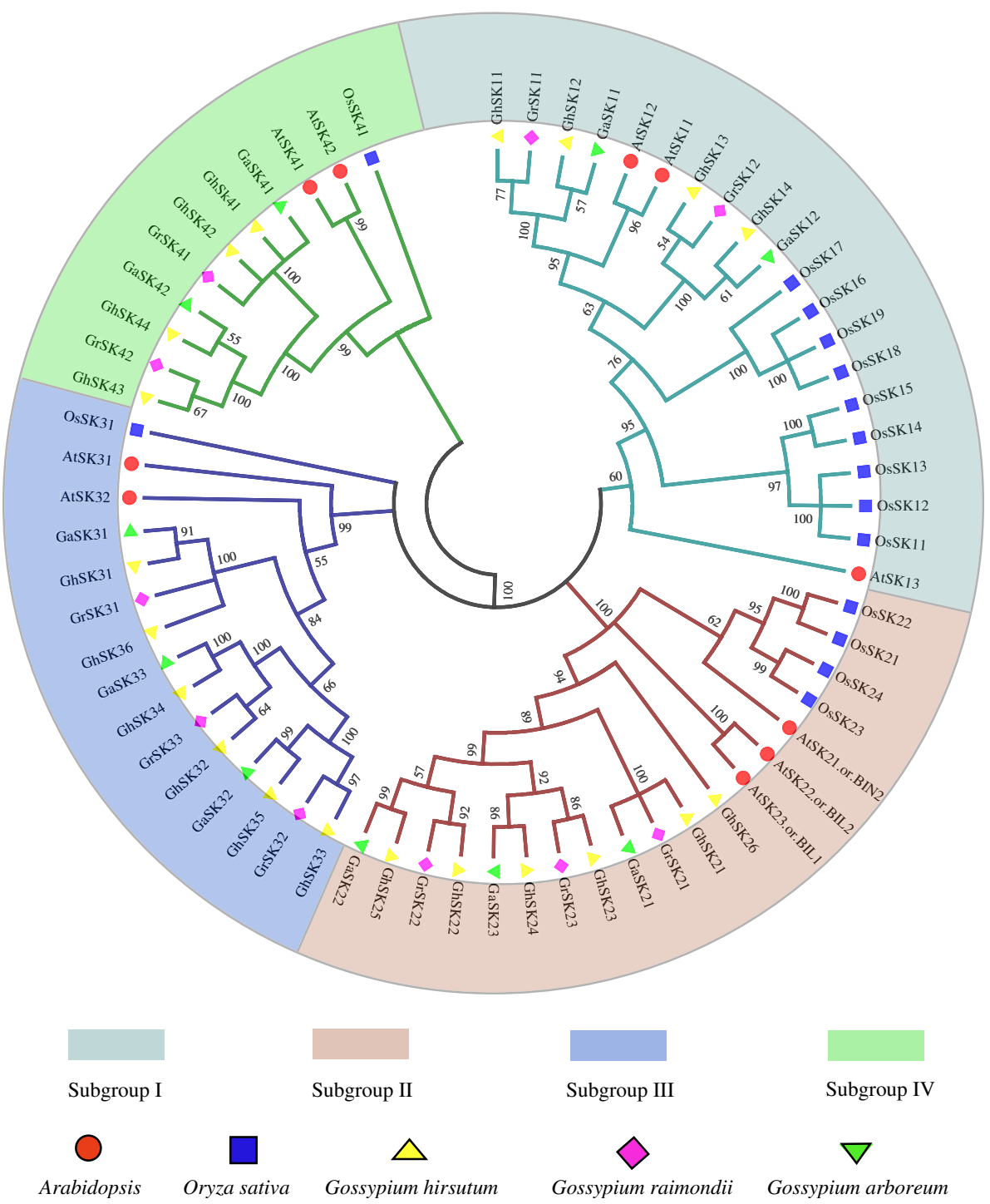

Fig. 1 Phylogenetic analysis of GSK3 proteins in Arabidopsis, rice, and species of cotton. 10 AtSKs, 15 OsSKs, 20 GhSKs, 10 GaSKs, and 10 GrSKs are divided into four clades. The four clades are respectively colored in cyan, reddish brown, violet, and green

study, 26, 28 and 13 conserved non-nested syntenic blocks around GSK genes were predicted between the genomes of G.hirsutum and G. arboreum, G. hirsutum and G. raimondii, and G. arboreum and G. raimondii, respectively (Fig. 4). The detailed information regarding each syntenic block is provided in Additional file 6: Table S3. The G. hirsutum genes on chromosomes A01, A08, A09, A11, A12, D14, D19, D21, D24, and D25 as well as scaffold 1340_A06, 3045_A11, and 4332_D22 were likely derived from conserved blocks on G. arboreum chromosome Ga02, Ga06, Ga08, Ga09, Ga11, and Ga12. Meanwhile, genes on G. raimondii chromosome Gr02, Gr04, Gr06, Gr07, Gr08, and Gr10 were predicted to possess ortholog genes on G. hirsutum chromosomes as well as orthologs of GaSKs.
GhSK expression profiles in various tissues and organs

Different tissue- and organ-specific gene expression patterns might be an indicator of distinct biological functions. Heat maps of transcriptomic data from the NCBI database revealed that most of the genes assigned to the same subfamily were clustered together and had similar expression profiles (Fig. 5). For example, most of GhSK genes were expressed at relatively low levels in 25,35 dpa ovules and fiber tissues. The exception being subfamily IV members, as well as GhSK33 and GhSK35 in 10 and 20 dpa ovule, GhSK34 in early stage fiber and GhSK32 in 5, 10, and 20 dpa fiber tissues where their expression was often significant. Most of GhSK genes had relatively high expression levels in $-1 \sim 20$ dpa ovules. Additionally, GhSK32, GhSK33, GhSK34, and GhSK35 
a G.hirsutum

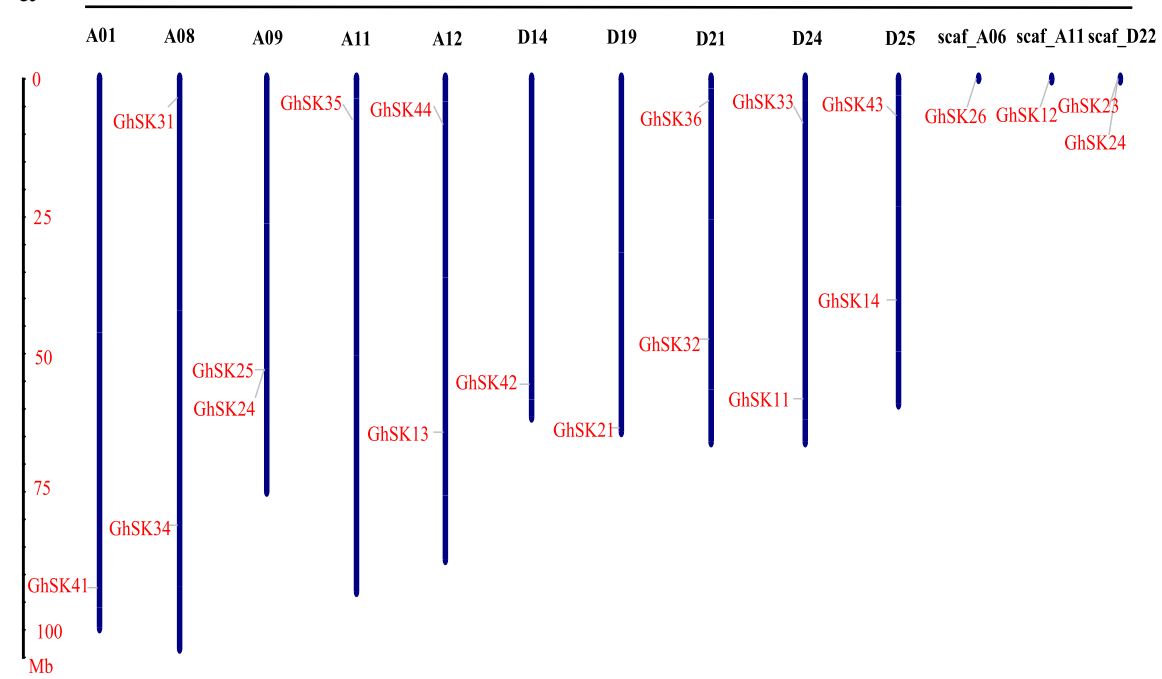

$\mathrm{b}$

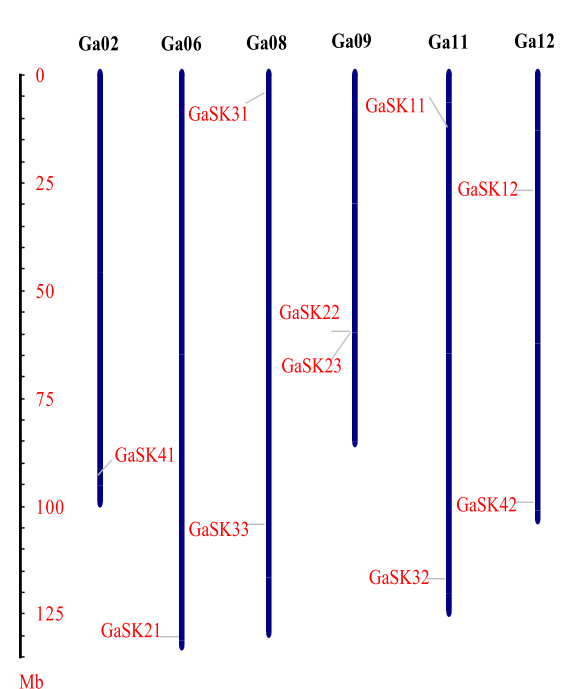

$\mathrm{c}$

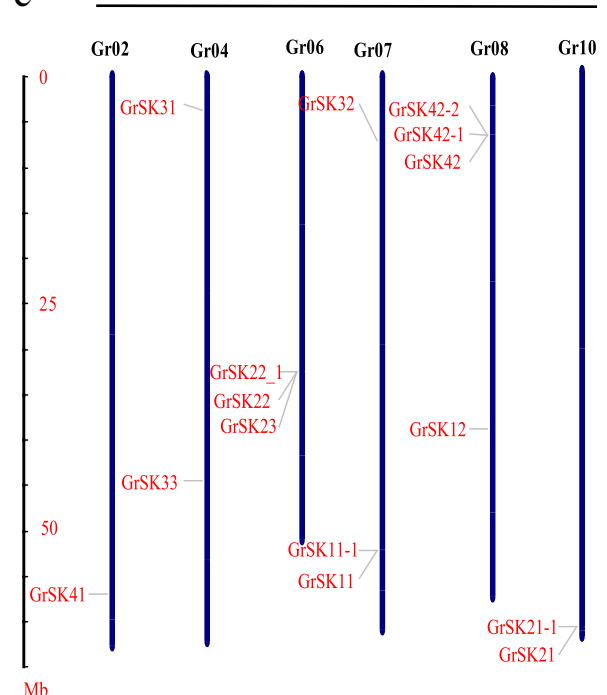

Fig. 2 Chromosomal assignment of GhSKs (a), GaSKs (b) and GrSKs (c). The relative chromosomal sizes (unit, Mb) of G. hirsutum, G. arboreum and G. raimondii were calculated from the published genome data of the kinds of cotton

were highly expressed in petal and stamen tissues. Apart from subfamily IV members, other GhSKs had relatively high, but uneven expression patterns in various floral organs. Most of the GhSK members showed poor expressions in the dry seed, while some exhibited slightly higher expression in the calycle (epicalyx). Nearly all the GhSKs had relatively high expression levels in leaf and stem, excluding GhSK31, GhSK33, GhSK35, GhSK36 and members of subfamily IV. Furthermore, only GhSK14, GhSK21, GhSK25, GhSK42, GhSK43 exhibited relatively high expression levels in root tissue.

The different members of the same gene family can exhibit diverse expression patterns in various tissues/organs and play different physiological functions [61]. To further validate the transcriptomic data, the expression levels of 20 GhSK genes were analyzed in 10 different tissues/organs, including flower, ovule (1,3, and $5 \mathrm{dpa})$, and fiber (7, 10, 15, and $20 \mathrm{dpa}$ ) by Q-PCR (Fig. 6). Here, 13 of the 20 genes (GhSK12, 13, 14, 22, 23, 24, 25, $32,35,41,42,43$, and 44 ) were preferentially or relatively highly expressed in different fiber development stages. The GSK gene expression was lower in whole flower tissues than in the isolated growing fibers.

\section{Analysis of GSK promoter regions}

Abundant hormone- and stress-related cis-elements, including cis-elements that confer high transcript levels and MYB-binding sites were detected in the regions $2 \mathrm{~kb}$ 


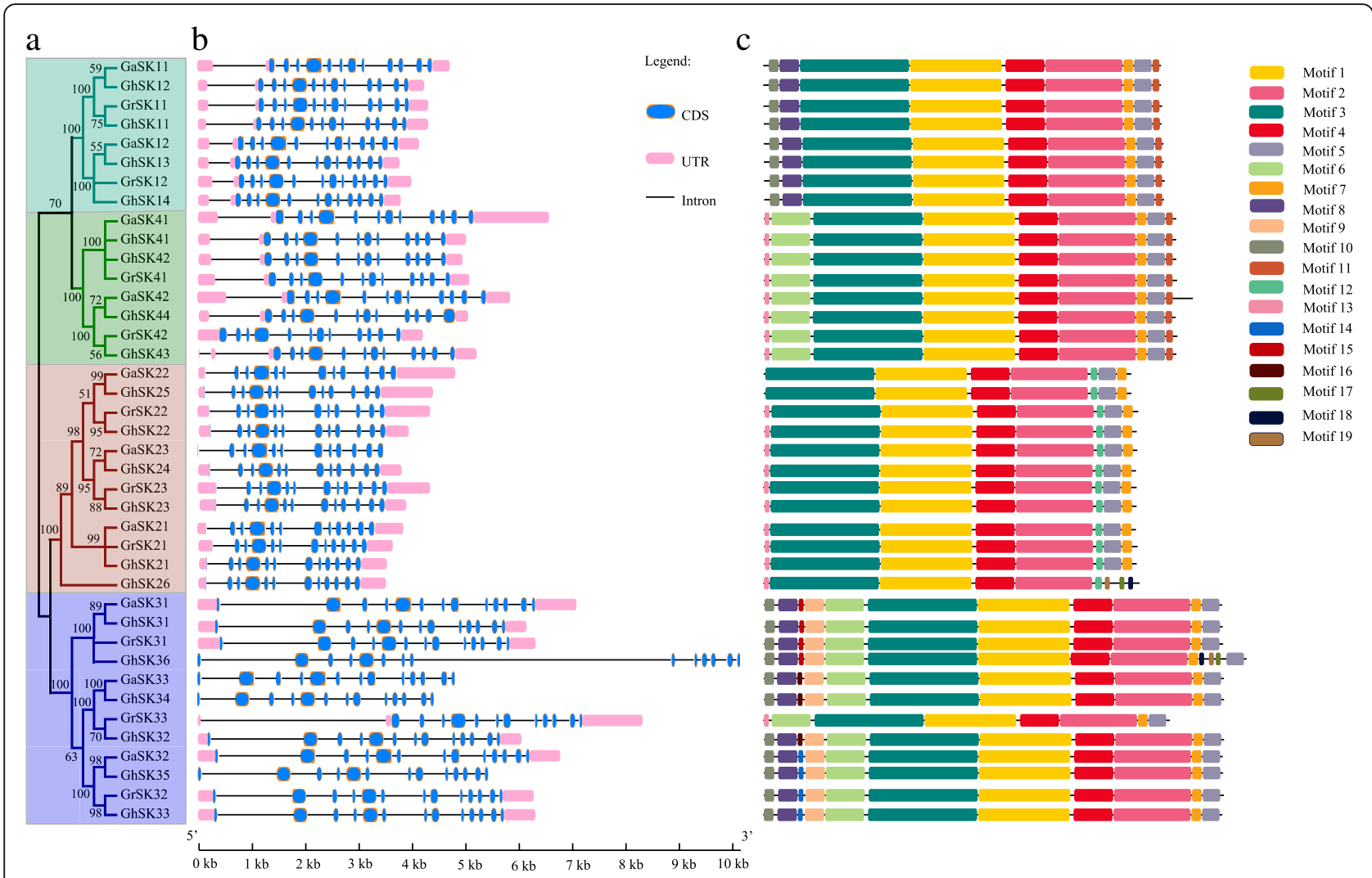

Fig. 3 Phylogenetic relationships between the cotton GSKs constructed using the Neighbor-Joining algorithm and Poisson model (a), gene structure predictions (b) and conversed protein motifs (c)

upstream of the transcription start site of different GhSK genes (Additional file 7: Figure S4 and Additional file 8: Table S4). The phylogenetically similar genes shared identical cis-elements. All of the GhSK promoter regions contained more than one cis-element that confers high transcript level. Additionally, heat-, defense-, stress-, salicylic acid-, and auxin-responsive cis-elements were identified in the promoter regions of almost all GhSK genes, except for GhSK44 (no heat-responsive element), GhSK13 and 32 (no defense- and stress-responsive elements), GhSK13, 14 and 43 (no salicylic acid-responsive element), and GhSK11, 12, 43, and 44 (no auxin-responsive element). Furthermore, wound-responsive elements were detected only in the promoter regions of GhSK21, GhSK26, and GhSK32, while only GhSK23 and 33 contained an ABA-responsive element. Moreover, 9 and 13 out of 20 GhSK genes contained low temperature- and GA-responsive elements respectively, while methyl jasmonate- and fungal elicitor-responsive cis-element were observed in 8 and 9 genes respectively. MYB-binding site or MYB binding site involved in regulating flavonoid gene expression was observed in 4 GhSKs promoter regions, while elements of drought-inducible MYB binding site were observed in 13 GhSKs promoter regions. Additionally, the ethylene-responsive element was identified in 8
GhSKs promoter regions. Furthermore, three kinds of tissue-specific (shoot, meristem, and seed) elements were found in the promoter regions of some of the GhSK genes.

\section{GSK gene expression patterns in response to several abiotic stresses}

To explore the physiological and functional relevance of GhSK genes, we investigated the expression patterns of 20 GhSK genes under different environmental stresses [i.e., Cold $\left(4^{\circ} \mathrm{C}\right)$, salinity, simulated drought (PEG), and heat $\left(38^{\circ} \mathrm{C}\right)$ ] (Fig. 7$)$. Among the 20 genes, expression levels of six GhSK (i.e., GhSK14, 22, 35, 41, 43, and 44) genes were down-regulated by all imposed stresses. Not all GhSK genes were induced or repressed by temperature stresses. Among them, GhSK21, 24, 26, and 36 were cold induced, GhSK21, 24 and 42 were up-regulated by heat stress, while the expression levels of GhSK31, 33, and 36 were repressed under heat condition. Except for GhSK25, 31, and 33, the expression levels of the other 11 GhSK genes (excluded those six uniformly down-regulated genes mentioned above) were up-regulated on exposure to PEG. GhSK11, 12, and 13 were slightly induced by PEG treatment during the first $3 \mathrm{~h}$. GhSK21, GhSK24, and GhSK26 were highly 
Table 2 Duplicated GSK gene pairs identified in G. hirsutum, G. arboreum, and G. raimondii

\begin{tabular}{|c|c|c|c|c|c|c|}
\hline \multirow[b]{2}{*}{ Clade } & \multicolumn{6}{|l|}{ Duplicates } \\
\hline & Gene ID & Gene name & Position & Gene ID & Gene name & Position \\
\hline$\|$ & Ga09G0899 & GaSK22 & Ga09: 59971361-59,974,663 & & & Ga09: 59971879-59,974,663 \\
\hline$\|$ & Ga09G0899 & GaSK22 & Ga09: 59971361-59,974,663 & & & Ga09: 59989139-59,991,790 \\
\hline$\|$ & Ga09G0899 & GaSK22 & Ga09: 59971361-59,974,663 & & & Ga06: 130211141-130,213,614 \\
\hline$\|$ & Ga09G0900 & GaSK23 & Ga09: 59988594-59,991,790 & & & Ga09: 59989139-59,991,790 \\
\hline$\|$ & Ga09G0900 & GaSK23 & Ga09: 59988594-59,991,790 & & & Ga09: 59971879-59,974,663 \\
\hline$\|$ & Ga09G0900 & GaSK23 & Ga09: 59988594-59,991,790 & & & Ga06: 130211141-130,213,614 \\
\hline IV & Ga02G1328 & GaSK41 & Ga02:91572951-91,576,357 & & & Ga12: 98906373-98,909,799 \\
\hline$\|$ & Ga06G2433 & GaSK21 & Ga06:130210718-130,213,627 & & & Ga09: 59989139-59,991,777 \\
\hline$\|$ & Ga06G2433 & GaSK21 & Ga06:130210718-130,213,627 & & & Ga09: 59971879-59,974,650 \\
\hline IV & Ga12G2665 & GaSK42 & Ga12:98904726-98,909,799 & & & Ga02: 91572950-91,576,342 \\
\hline$\|$ & Gorai.010G241600.3 & GrSK21 & Gr10: 61087060-61,090,705 & & & Gr10: 61087549-61,090,443 \\
\hline$\|$ & Gorai.010G241600.2 & GrSK21-1 & Gr10: 61085419-61,090,069 & & & Gr10: 61087549-61,090,014 \\
\hline III & Gorai.004G042400.1 & GrSK31 & Gr04: 3659148-3,665,477 & & & Gr04: 3659643-3,665,066 \\
\hline III & Gorai.004G156700.2 & GrSK33 & Gr04: 44445510-44,453,846 & & & Gr04: 44449136-44,452,709 \\
\hline III & Gorai.007G096100.1 & GrSK32 & Gr07: 7076952-7,083,244 & & & Gr07: 7077235-7,082,655 \\
\hline | & Gorai.007G308500.3 & GrSK11-1 & Gr07: 52192786-52,196,322 & & & Gr07: 52193148-52,195,963 \\
\hline । & Gorai.007G308500.1 & GrSK11 & Gr07: 52192786-52,197,103 & & & Gr07: 52193148-52,195,963 \\
\hline IV & Gorai.002G218100.1 & GrSK41 & Gr02: 56980865-56,985,951 & & & Gr02: 56982189-56,985,586 \\
\hline IV & Gorai.002G218100.1 & GrSK41 & Gr02: 56980865-56,985,951 & & & Gr08: 6199127-6,202,516 \\
\hline IV & Gorai.008G045800.2 & GrSK42-2 & Gr08: 6198649-6,203,828 & & & Gr02: 56982189-56,985,579 \\
\hline IV & Gorai.008G045800.2 & GrSK42-2 & Gr08: 6198649-6,203,828 & & & Gr08: 6199120-6,202,516 \\
\hline IV & Gorai.008G045800.3 & GrSK42-1 & Gr08: 6198649-6,203,828 & & & Gr02: 56982189-56,985,579 \\
\hline IV & Gorai.008G045800.3 & GrSK42-1 & Gr08: 6198649-6,203,828 & & & Gr08: 6199120-6,202,516 \\
\hline IV & Gorai.008G045800.6 & GrSK42 & Gr08: 6198704-6,203,828 & & & Gr02: 56982189-56,985,579 \\
\hline IV & Gorai.008G045800.6 & GrSK42 & Gr08: 6198704-6,203,828 & & & Gr08: 6199120-6,202,516 \\
\hline | & Gorai.008G136600.1 & GrSK12 & Gr08: 38585110-38,589,107 & & & Gr08: 38585874-38,588,662 \\
\hline$\|$ & Gorai.006G089300.1 & GrSK22 & Gr06: 32530678-32,535,037 & & & Gr06: 32531407-32,534,186 \\
\hline$\|$ & Gorai.006G089300.1 & GrSK22 & Gr06: 32530678-32,535,037 & & & Gr06: 32547582-32,550,218 \\
\hline$\|$ & Gorai.006G089300.2 & GrSK22-1 & Gr06: 32530521-32,535,037 & & & Gr06: $32531410-32,534,186$ \\
\hline$\|$ & Gorai.006G089300.2 & GrSK22-1 & Gr06: 32530521-32,535,037 & & & Gr06: 32547583-32,550,218 \\
\hline$\|$ & Gorai.006G089400.1 & GrSK23 & Gr06: 32546669-32,551,023 & & & Gr06: 32531410-32,534,186 \\
\hline$\|$ & Gorai.006G089400.1 & GrSK23 & Gr06: 32546669-32,551,023 & & & Gr10: 61087562-61,089,975 \\
\hline IV & Gh_A12G0411 & GhSK44 & A12: 8244999-8,248,430 & Gh_D01G1809 & GhSK42 & D14: 55472542-55,475,929 \\
\hline IV & Gh_A12G0411 & GhSK44 & A12: 8244999-8,248,430 & Gh_A01G1558 & GhSK41 & A01: 92410496-92,413,866 \\
\hline IV & Gh_A12G0411 & GhSK44 & A12: 8244999-8,248,430 & Gh_D12G0407 & GhSK43 & D25: 6533936-6,537,332 \\
\hline$\|$ & Gh_D06G2142 & GhSK21 & D19: 63261021-63,263,915 & Gh_A06G2020 & GhSK26 & Scaf1340_A06: 43051-45,948 \\
\hline । & Gh_D11G2830 & GhSK11 & D24: 58071261-58,074,075 & Gh_A11G3270 & GhSK12 & Scaf3045_A11: 160891-163,705 \\
\hline III & Gh_A08G0285 & GhSK31 & A08: 3329052-3,334,479 & Gh_D08G0378 & GhSK36 & D21: 3871235-3,880,133 \\
\hline IV & Gh_D12G0407 & GhSK43 & D25: 6533937-6,537,332 & Gh_D01G1809 & GhSK42 & D14: 55472542-55,475,929 \\
\hline IV & Gh_D12G0407 & GhSK43 & D25: 6533937-6,537,332 & Gh_A01G1558 & GhSK41 & A01: 92410496-92,413,866 \\
\hline IV & Gh_D12G0407 & GhSK43 & D25: 6533937-6,537,332 & Gh_A12G0411 & GhSK44 & A12: $8244998-8,248,430$ \\
\hline III & Gh_D08G0378 & GhSK36 & D21: 3871236-3,880,133 & Gh_A08G0285 & GhSK31 & A08: $3329051-3,334,479$ \\
\hline | & Gh_D12G1230 & GhSK14 & D25: 40170076-40,172,846 & Gh_A12G1106 & GhSK13 & A12: 64114145-64,116,909 \\
\hline
\end{tabular}


Table 2 Duplicated GSK gene pairs identified in G. hirsutum, G. arboreum, and G. raimondii (Continued)

\begin{tabular}{|c|c|c|c|c|c|c|}
\hline \multirow[b]{2}{*}{ Clade } & \multicolumn{6}{|l|}{ Duplicates } \\
\hline & Gene ID & Gene name & Position & Gene ID & Gene name & Position \\
\hline IV & Gh_D01G1809 & GhSK42 & D14: 55472543-55,475,936 & Gh_A01G1558 & GhSK41 & A01: 92410496-92,413,873 \\
\hline IV & Gh_D01G1809 & GhSK42 & D14: 55472543-55,475,936 & Gh_D12G0407 & GhSK43 & D25: 6533943-6,537,332 \\
\hline$\|$ & Gh_A09G0712 & GhSK25 & A09: 52841598-52,844,894 & & & A09: 52842113-52,844,894 \\
\hline ॥ & Gh_A09G0712 & GhSK25 & A09: 52841598-52,844,894 & Gh_A09G0713 & GhSK24 & A09: 52861923-52,864,569 \\
\hline$\|$ & Gh_A09G0712 & GhSK25 & A09: 52841598-52,844,894 & Gh_D09G2469 & GhSK22 & Scaf4332_D22: 137948-140,717 \\
\hline$\|$ & Gh_A09G0712 & GhSK25 & A09: 52841598-52,844,894 & Gh_D09G2468 & GhSK23 & Scaf4332_D22: 123123-125,754 \\
\hline$\|$ & Gh_A09G0713 & GhSK24 & A09: 52861382-52,864,569 & & & A09: 52842113-52,844,894 \\
\hline$\|$ & Gh_A09G0713 & GhSK24 & A09: 52861382-52,864,569 & & & A09: 52861923-52,864,569 \\
\hline$\|$ & Gh_A09G0713 & GhSK24 & A09: 52861382-52,864,569 & Gh_D09G2469 & GhSK22 & Scaf4332_D22: 137948-140,717 \\
\hline$\|$ & Gh_A09G0713 & GhSK24 & A09: 52861382-52,864,569 & Gh_D09G2468 & GhSK23 & Scaf4332_D22: 123123-125,754 \\
\hline III & Gh_D11G0907 & GhSK33 & D24: 7839230-7,844,650 & Gh_A11G0778 & GhSK35 & A11: 7649122-7,654,545 \\
\hline III & Gh_A08G1158 & GhSK34 & A08: 81021333-81,025,764 & Gh_D08G1440 & GhSK32 & D21: 47361119-47,366,590 \\
\hline IV & Gh_A01G1558 & GhSK41 & A01: 92410497-92,413,873 & Gh_D01G1809 & GhSK42 & D14: 55472542-55,475,936 \\
\hline IV & Gh_A01G1558 & GhSK41 & A01: 92410497-92,413,873 & Gh_D12G0407 & GhSK43 & D25: 6533943-6,537,332 \\
\hline III & Gh_A11G0778 & GhSK35 & A11: 7649123-7,654,545 & Gh_D11G0907 & GhSK33 & D24: 7839229-7,844,650 \\
\hline III & Gh_D08G1440 & GhSK32 & D21: 47361120-47,366,590 & Gh_A08G1158 & GhSK34 & A08: 81021332-81,025,764 \\
\hline । & Gh_A12G1106 & GhSK13 & A12: 64114146-64,116,909 & Gh_D12G1230 & GhSK14 & D25: 40170075-40,172,846 \\
\hline$\|$ & Gh_A06G2020 & GhSK26 & Scaf1340_A06: 43052-45,877 & Gh_D06G2142 & GhSK21 & D19: 63261091-63,263,915 \\
\hline । & Gh_A11G3270 & GhSK12 & Scaf3045_A11: 160892-163,705 & Gh_D11G2830 & GhSK11 & D24: 58071260-58,074,075 \\
\hline$\|$ & Gh_D09G2468 & GhSK23 & Scaf4332_D22: 123124-126,295 & & & A09: 52861923-52,864,569 \\
\hline$\|$ & Gh_D09G2468 & GhSK23 & Scaf4332_D22: 123124-126,295 & & & A09: 52842113-52,844,894 \\
\hline$\|$ & Gh_D09G2468 & GhSK23 & Scaf4332_D22: 123124-126,295 & Gh_D09G2469 & GhSK22 & Scaf4332_D22: 137948-140,717 \\
\hline$\|$ & Gh_D09G2469 & GhSK22 & Scaf4332_D22: 137949-141,233 & & & A09: 52861923-52,864,569 \\
\hline ॥ & Gh_D09G2469 & GhSK22 & Scaf4332_D22: 137949-141,233 & & & A09: 52842113-52,844,894 \\
\hline$\|$ & Gh_D09G2469 & GhSK22 & Scaf4332_D22: 137949-141,233 & Gh_D09G2468 & GhSK23 & Scaf4332_D22: 123123-125,754 \\
\hline
\end{tabular}

responsive to PEG treatments, with 3-fold, 2-fold, and 4-fold increases, respectively, irrespective of different times after treatment. Unlike GhSK31 and 33, expression levels of the other GhSK3s exhibited more than 2-fold increases at $3 \mathrm{~h}$ after PEG treatment excluding GhSK36 that peaked at $1 \mathrm{~h}$ with about 4-fold changes. The expression level of GhSK 42 was only slightly affected by PEG at $1 \mathrm{~h}$ but was subsequently induced, reaching its peak level (approximately 2.5-fold increase) at $3 \mathrm{~h}$. For salinity stress, expression levels of GhSK11, 25, and 42 were not significantly induced at any time point. However, GhSK12, 24, and 32 expression levels increased during the first $3 \mathrm{~h}$ and then decreased slightly. Additionally, the expression levels of GhSK13, 23, 31, 33 and 36 were up-regulated over the first $6 \mathrm{~h}$ (approximately 3-fold, 3-fold, 3-fold, 2.2-fold, and 2.3-fold increases, respectively) and then decreased. Meanwhile, GhSK21, 26, 34 as well as 42 expression levels were up-regulated gradually and finally peaked at $12 \mathrm{~h}$ after salinity treatment.

\section{Regulatory sub-networks involving GhSK and other G. hirsutum genes}

Co-expressed genes exhibit similar increases or decreases in transcription levels in different samples [62, 63]. The GSKs are known to influence several developmental, signaling, and physiological processes in plants [2]. The co-expression between GhSK and other G. hirsutum genes were analyzed using the R package (WGCNA), and the co-expressed genes linking to other GhSK genes (GhSKs linkers) were identified and annotated (Fig 8, Additional file 9: Figure S5a, S5b, Additional file 10: Figure S6 and Additional file 11: Table S5.1, S5.2 and S5.3). We detected five modules (blue, brown, green, turquoise and yellow), in which nine GhSK genes were co-expressed with other G. hirsutum genes. Surprisingly, the GhSK32 (found in brown module) was co-expressed with $2781 \mathrm{G}$. hirsutum genes across all the analyzed samples (Additional file 10: Figure S6 and Additional file 11: Table S5.1, S5.2, and S5.3). The GhSK34 (also present in the brown module) had 2142 co-expressed genes (Additional file 10: 


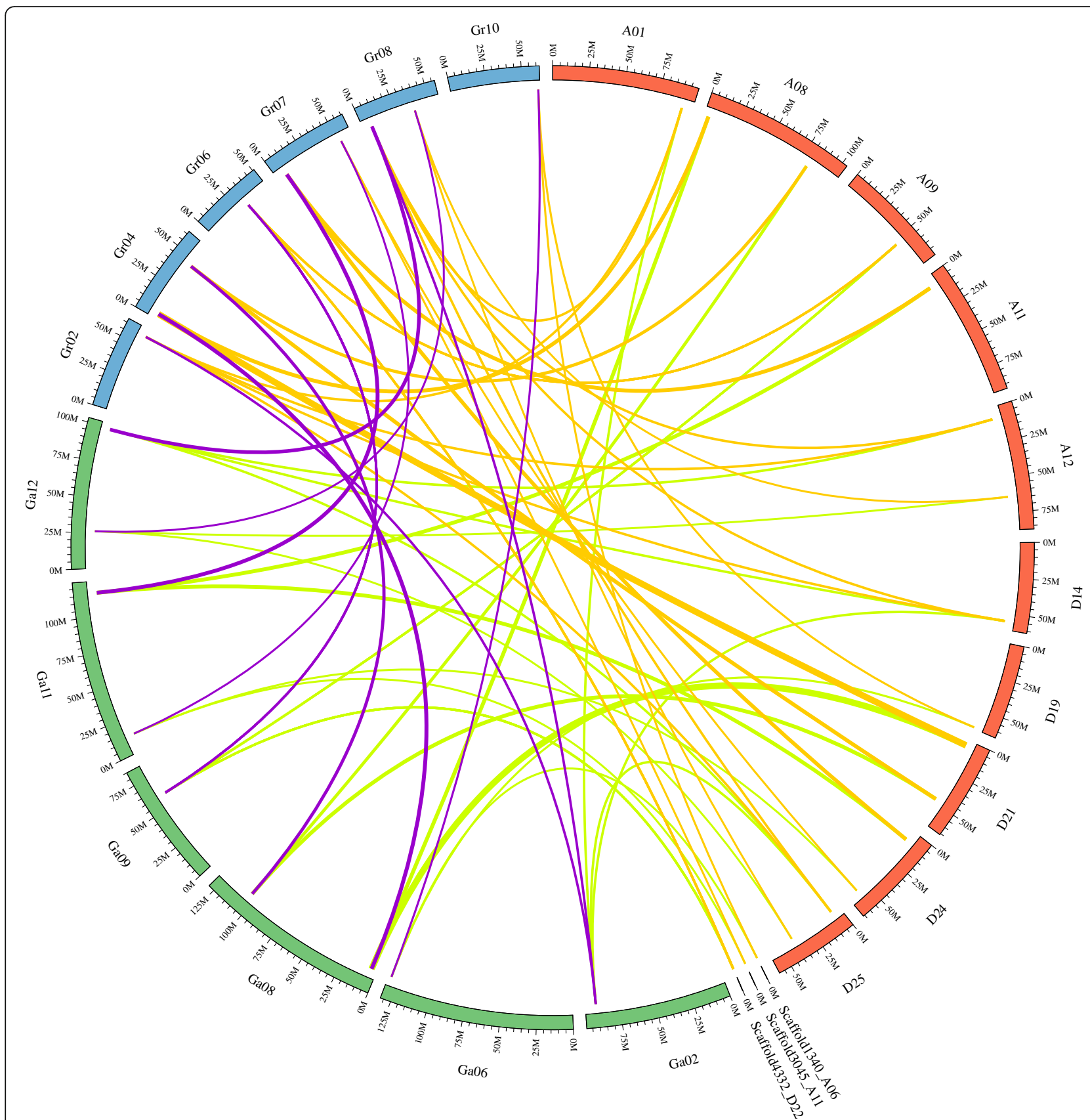

Fig. 4 Orthologous GSK genes identified in G. hirsutum, G. arboreum and G. raimondii. Links between G. hirsutum and G. arboreum are colored in cyan, G. hirsutum and G. raimondii in orange, G. arboreum and G. raimondii in light purple. Chromosomes of G. hirsutum, arboreum and raimondii were respectively colored in orange-red, green and blue

Figure S6 and Additional file 11: Table S5.3). These genes in the brown module were enriched in $55 \mathrm{GO}$ terms (FDR $<0.05$ ) (i.e., 30, 20, and 5 related to molecular functions, biological processes, and cellular components, respectively). Additionally, GhSK13 was co-expressed with 17 genes (including GhSK14) (Fig. 8a), similarly, GhSK31 was co-expressed with 20 genes (Fig. 8b). GhSK11, GhSK25, and GhSK24 were co-expressed with 19, 1 (Gh_A04G1034), and 20 other G. hirsutum genes, respectively (Fig. 8c), meanwhile, GhSK42 and GhSK41 were co-expressed with 6 and 11 genes, respectively (Fig. 8d). Among these genes, GhSK11 and GhSK24 were indirectly co-expressed with each other, as were GhSK42 and GhSK4 (directly/indirectly). Similarly, GhSK32 co-expressed with GhSK34 both directly and indirectly (Additional file 10: Figure S6 and Additional file 11: Table S5.3). The co-expressed genes in the brown module were mainly enriched (Top20 GO) for protein phosphorylation, protein kinase activity, integral 


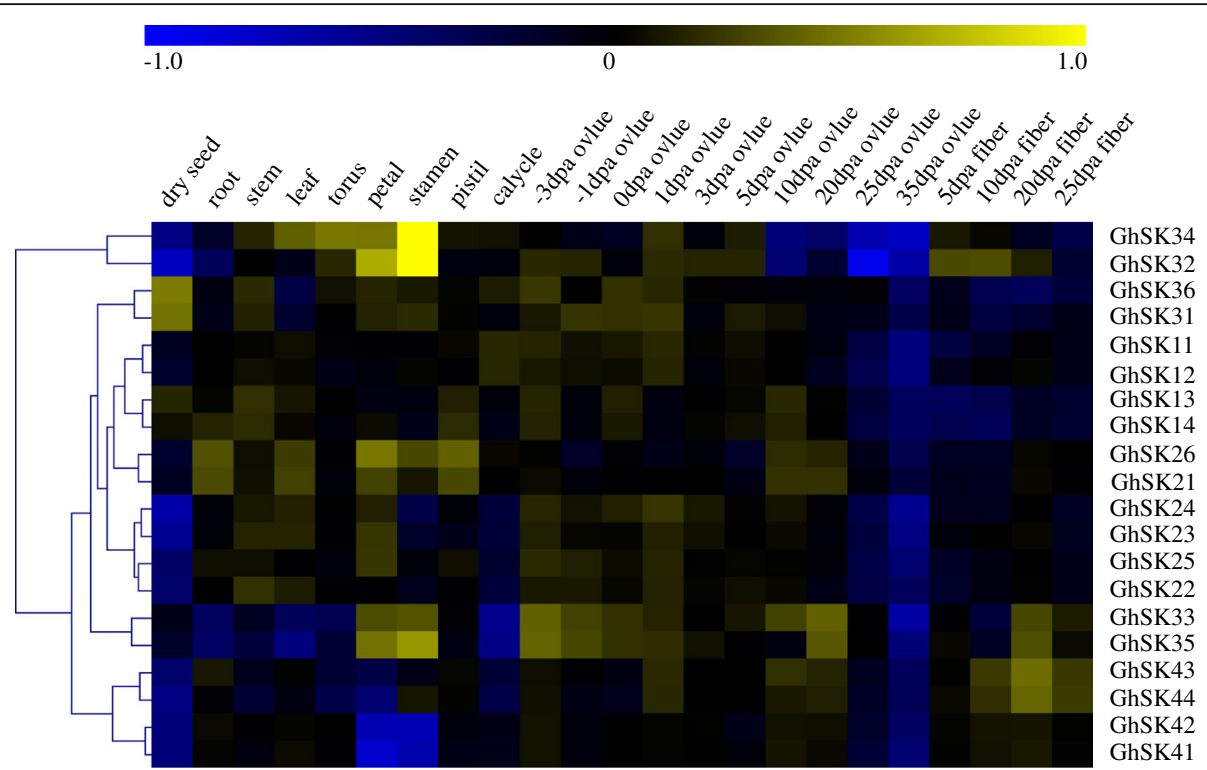

Fig. 5 Hierarchical clustering of gene expression profiles of all GhSK members in 23 diverse organs/tissues (dry seed, root, stem, leaf, torus (receptacle), petal, stamen, pistil, calycle (epicalyx), 10 stages of ovule and 4 stages of fiber). The FPKM values, log 10-transformed, were utilized to generate the heat map. The blue colors indicate low expression levels, while yellow represents high expression levels

component of membrane, Calcium ion binding, transmembrane transport, and enzyme inhibitor activity (Additional file 9: Figure S5a). Further, these were also significantly enriched in pentose and glucoronate interconversion, plant-pathogen interaction, as well as starch and sucrose metabolism pathways (Top20 KEGG) (Additional file 9: Figure S5b). The GO (FDR $<0.05)$ and KEGG $(p<0.05)$ enrichment results for the other target genes are presented in Additional file 11: Table S5.1 and S5.2.

\section{Discussion}

Protein kinases form a large family of enzymes that mediate eukaryotic cell responses to external stimuli [64]. To date, several protein kinase family members have been identified, including GSK3, MAPKKK, Pto-like protein kinase, and PP2C [65-68]. Among these enzymes, GSK3 reportedly regulates different physiological and developmental processes in mammals and plants $[2,9,45,69,70]$. However, the genome-wide characterization of GSK3-like kinases has been limited to model plants, even though these enzymes were first reported decades ago [67].

\section{The cotton GSK gene family expanded but remained highly conserved during evolution}

The availability of high-quality whole draft genome sequences has enabled the characterization of G. aboreum, $G$. raimondii, and $G$. hirsutum genes. Interestingly, the number of G. hirsutum GSK genes almost equals to the sum of G. arboreum and G. raimondii genes, possibly because of its formation as an allotetraploid following hybridization of $\mathrm{A}$ and $\mathrm{D}$ genome progenitors [33, 71]. Consistent with the names of corresponding A. thaliana and rice genes [45], we renamed the cotton GSK genes as GhSKs (i.e., G. hirsutum Shaggy/GSK3-like kinases), GaSKs and GrSKs (Table 1). Additionally, the cotton GSK genes were divided into four subfamilies in accordance with the $A$. thaliana and rice GSK genes. We observed that subfamily II was the largest containing 14 members, then subfamily III with 12 members, IV and I comprised of 10 and 9 members respectively (Table 1 and Fig. 1). Moreover, the sub-cellular location of almost all GSKs was predicted to be in the nucleus, membrane and cytoplasm, except for GaSK23 (cytoplasmic) (Table 1 and Fig. 1). Additionally, the kinase domains of AtSKs and OsSKs were 65-72\% similar to human GSK3 $\beta$ [45]. Here, we aligned the peptide sequences of AtSKs, OsSKs, GaSKs, GrSKs, and GhSKs. All of these GSKs contain related conserved domains and residues, suggesting these enzymes are highly conserved across species and that of the GSK family existed before the separation of these different species [2].

Consistent with the AtSKs, cotton GSKs are clustered into four clades [45]. The results of phylogenetic analysis and the presence of conversed domains suggested that the GSKs may have analogous biological functions [2]. However, various bootstrap values in the phylogenetic tree were low, potentially because of a relatively low similarity in the protein sequences of different species. This implied there was considerable variability in the GSK gene sequences, even though the protein motifs were highly conserved (Fig. 1). Furthermore, the cotton 


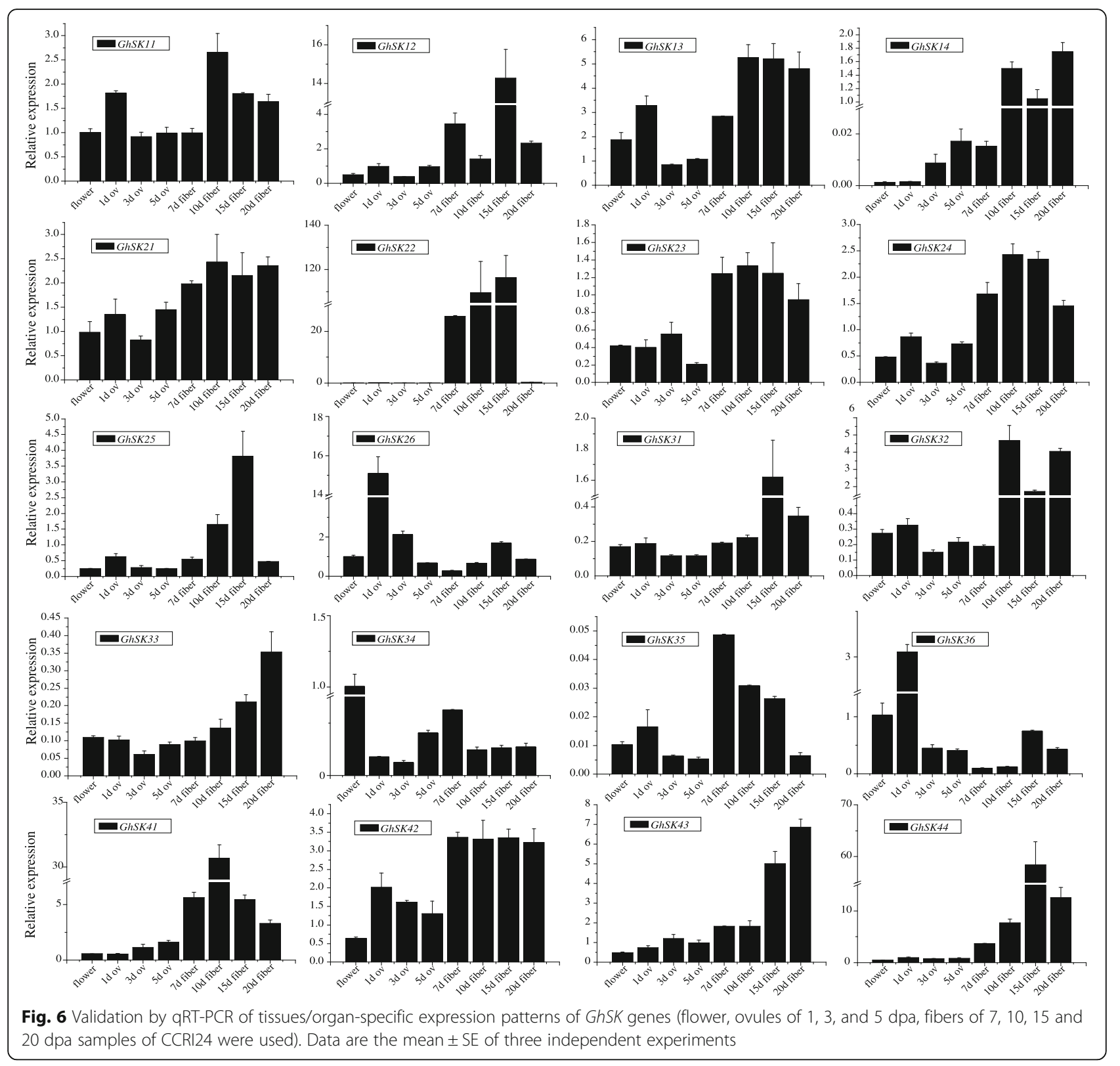

GSK genes clustered in the same subfamily were observed to encode proteins with similar motifs and protein architectures, suggesting the protein structures were conserved among members of the same subfamily. Nevertheless, there was significant diversity in the N-terminal domains of GSKs between different subfamilies, which was consistent with the findings of Forde and Dale [72]. Protein kinases are important for regulating protein phosphorylation in many cellular activities [73]. Integrating the results of conserved protein motifs, multiple sequence alignment, and previously published research [2], we speculate that a functional GSK kinase domain (motifs 1 to 4 ) was present in all cotton GSKs although we have not confirmed this enzymatically (Fig. 3c and Additional file 5: Figure S3). Additionally, most of these GSKs also contain serine and/or threonine kinase active sites and tyrosine residues, which could be phosphorylated by other kinases to modulate their enzymatic activity. This is believed to occur in all GSK3s and is required for the full kinase activity of human GSK3 3 . [74, 75].

\section{Gene duplicates and synteny blocks were detected} between cotton GSK genes

Allotetraploid cotton was generated from the fusion of two diploid cotton species, with chromosomes 1-13 and 14-26 derived from $\mathrm{A}$ and $\mathrm{D}$ genomes, respectively [33, 71]. In our study, GSK genes were unevenly distributed on the 


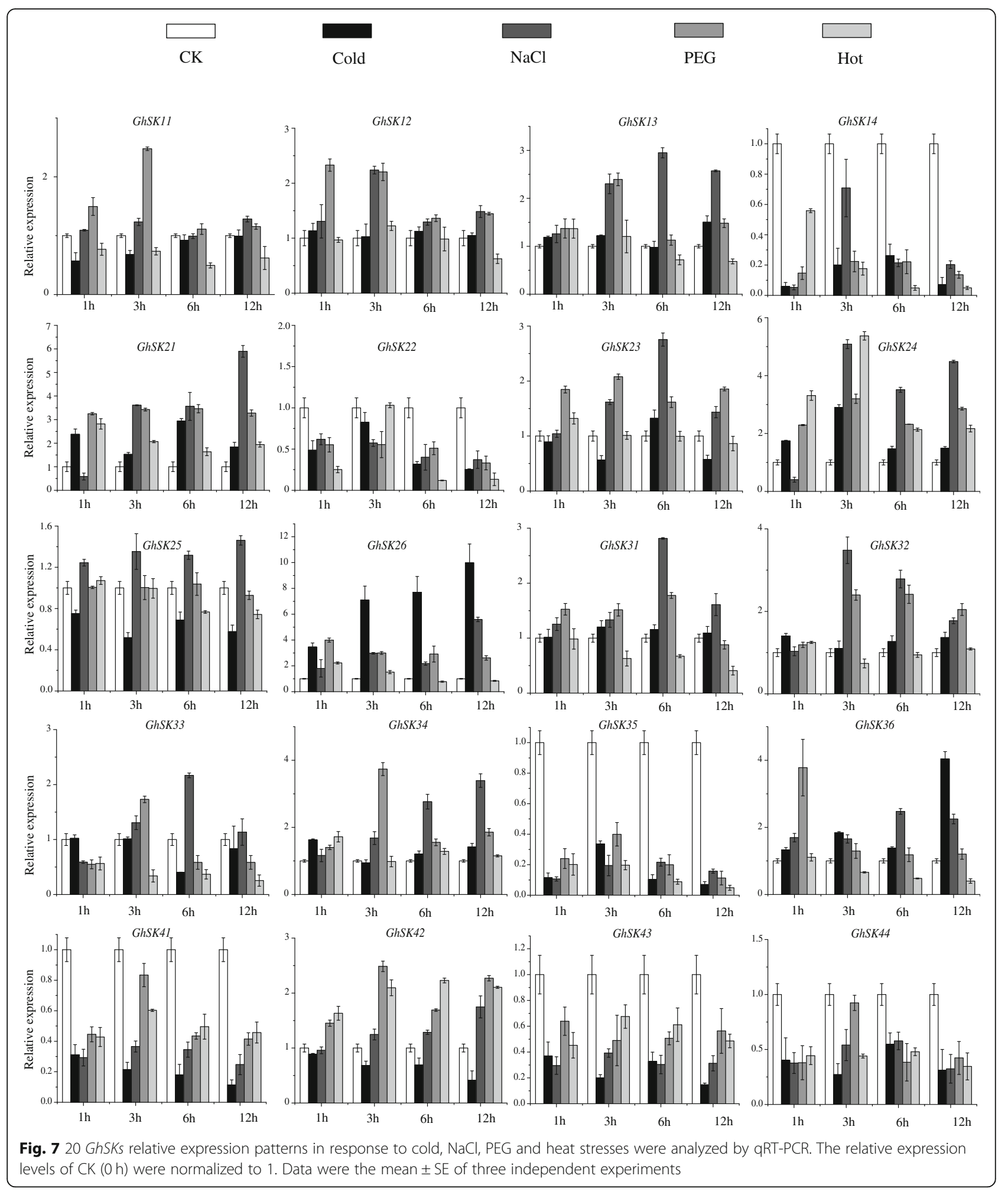

chromosomes of the three analyzed cotton species (diploid G. arboreum and G. raimondii as well as allotetraploid G. hirsutum). Thus, GSK gene duplication events might have occurred in these chromosomes during evolution, with neo- or sub-functionalization of these genes.
Orthologs are genes in different genomes that were derived from the same ancestral gene; they encode proteins with similar biological functions, whereas paralogs are defined as genes derived from a single gene via a duplication event, encoding proteins with different 


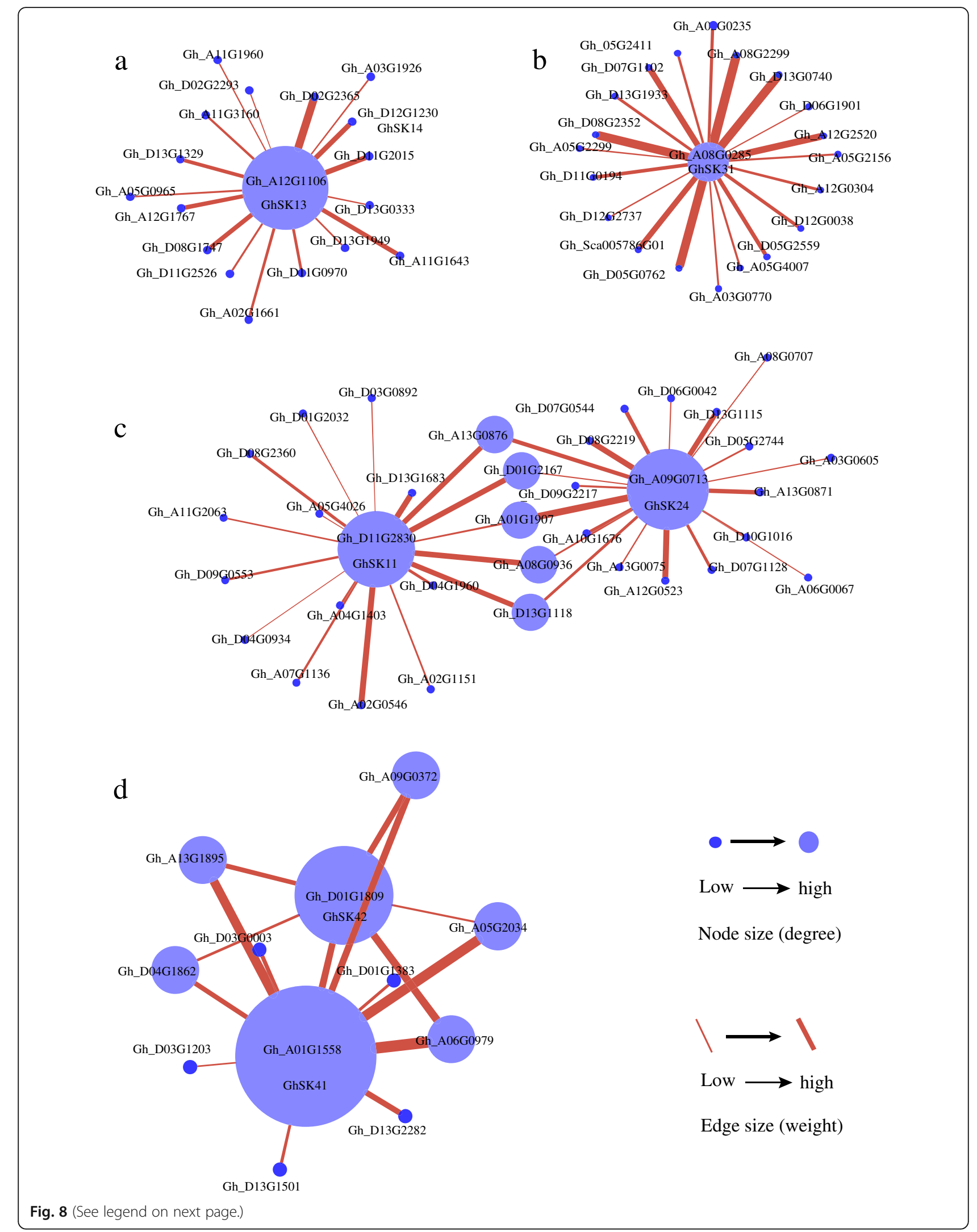


(See figure on previous page.)

Fig. 8 The weighted gene co-expression analysis modules of GhSK genes visualized by Cytoscape software. a Co-expression sub-network for GhSK13. b Co-expression sub-network for GhSK31. c Co-expression sub-network for GhSK14 and GhSK24. d Co-expression sub-network for GhSK42 and GhSK41. Nodes are genes with node size related to the number of connections of that gene to other co-expressed genes. Nodes are connected by lines (edges) with the weight of the line determined by the size of the correlation coefficient with thicker lines having a higher correlation and hence higher certainty

functions $[76,77]$. During evolutionary processes, new genes were generated as a result of large segmental and small-scale tandem duplications or polyploidization $[60,78]$. Duplicated genes are often found to be involved in the formation of paralogous genes present in gene families [78]. Tandem duplication usually results from unequal crossovers [79] and multiple occurrences of these can result in the expansion or reduction of different gene family members [60]. Here, G. arboreum, G. ramondii, and G. hirsutum contain more than one GSK gene clusters and genes within them with similar intron numbers suggesting that these duplicated genes might be the result of unequal crossovers. Additionally, recent studies demonstrated that the $G$. arboreum and $G$. raimondii genomes underwent at least two whole-genome duplication events [35, 36]. Although the total number of G. hirsutum genes dramatically expanded after polyploidization and duplication, gene loss also occurred during the evolution of upland cotton [39]. These might be the cause of the greater number of paralog gene pairs (25) in upland cotton compared to G. arboreum (10) and the uneven distribution of GSK genes on different chromosomes. Considering that orthologs might exhibit the same biological functions over the course of evolution [80], the syntenic blocks between G. hirsutum and G. arboreum, G. hirsutum and $G$. raimondii, and G. arboreum and G.raimondii were identified. All GhSK genes had at least one syntenic gene in G. arboreum and G. raimondii. Further, most of the highly similar gene pairs in regions around GSK genes found by comparing G. arboreum and G. hirsutum (red), G. raimondii and G. hirsutum (blue), and G. arboreum and G. raimondii (orange) in our study coincided with previous synteny analyses of various published cotton genomes [19, 34, 43], marked in different colors mentioned above and detailed in Additional file 6: Table. S3, suggesting that most of the cotton GSK loci were conserved during evolution.

\section{GhSKs are involved in fiber development and abiotic stresses response}

Variations in the expression patterns of GSK family members in various tissues/organs may be related to their functional differences [61]. To further characterize the potential regulatory functions of
GhSKs affecting cotton growth and development, the expression patterns of 20 GhSK genes were analyzed using both transcriptomic data and qRT-PCR data. Similar tissue- and organ-specific expression patterns were observed for the same GhSK gene based on either transcriptome or qRT-PCR data. Furthermore, GhSK22, 41, and GhSK44 were preferentially expressed in fibers. The GhSK12, 25, and 32 expression levels were also relatively high in different fiber tissues. GSK genes were previously reported to be involved in BR signaling in flowering plants [2]. These kinases may help to control cell elongation in plants because BRs regulate cell elongation [81]. However, gene expression levels of most GhSKs were not induced or repressed by exogenous BL treatment (Additional file 12: Figure S7). Previous reports indicated that brassinolide treatment reduced in vivo phosphorylation of BIN2, a special GSK3 member, and inhibited its function [82]. Similarly, BL treatment has been reported to lead to reductions in the protein accumulation and to inhibit the phosphorylation of AtSK12 [82]. The BR signal transduction pathway starts from a receptor kinase (BRI) to BR responsive transcription factors BZR1 and BES1, through a process of phosphorylation and dephosphorylation [83-85]. Last but not the least, we checked the differential expression levels of AtSKs in previously published transcriptome data of BL treated Arabidopsis [86], but similarly saw no evidence of transcriptional induction or repression of AtSKs. We speculate that BRs might affect GSKs at the protein and post-translational modification levels, rather than at the level of transcriptional regulation. Additionally, BIN2 functions in the crosstalk between BRs and auxin. AtSK21/BIN2 expression accelerates lateral root formation via the activation of ARF7 and ARF19, which are two factors involved in auxin-mediated lateral root development [87]. Meanwhile, TGA elements described as auxin-responsive elements were found in almost all GhSK gene promoter regions, and previous research has demonstrated that NAA (a synthetic auxin) enhances fiber elongation by suppressing secondary wall cellulose synthesis [88]. Furthermore, the regulation of auxin biosynthesis in ovule epidermal cells of cotton can enhance fiber quality and yield [89]. Additionally, ethylene was reported as playing a major role during fiber elongation [90], and ERE (ethylene responsive elements) were found in $8 \mathrm{GhSK}$ promoter regions. The current evidence suggests that GhSKs may therefore mediate fiber development and elongation. 
Plant responses to abiotic and biotic stresses are found to be regulated by GSK3 [2], although not in a consistent fashion [12, 15, 91-95]. Previous studies confirmed that GSK3 expression is up-regulated by salt stress in $A$. thaliana [12], rice [91], wheat [96], and sugarcane [95]. Moreover, the expressions of AtSK13, 31, and 42 are also up-regulated by osmotic stress, while OsGSK3 expression is down-regulated under drought conditions [91]. Consistent with these findings, we determined that the transcription of most GhSK genes were up-regulated in response to several abiotic stresses. The opposite trend was observed for six other GhSK genes. In contrast, GhSK25 expression was rarely affected under abiotic stress conditions (Fig. 7). Another study indicated that OsGSK3 expression is up-regulated on exposures to salt, cold, and drought stresses, mechanical injury, and exogenous ABA [6]. These results imply the encoded kinase activates signal cascade during stress. Besides, over-expressing TaSK5 cDNA in A. thaliana enhances the resistance of the transgenic plants to salinity and drought stresses, while their tolerance to freezing stress is retained [97]. Coupled with these results, we may conclude that stress-responsive cis-elements in the GhSK promoter regions play crucial roles during plant responses to various abiotic stresses.

\section{Several fiber-related genes are co-expressed with GhSKs}

Glycogen synthase kinase 3 is a serine/threonine kinase with several vital roles in animals [93]. There are about 18 proteins verified as direct substrates of GSK3. This kinase is associated with multiple molecular and cellular mechanisms affecting glycogen metabolism, cell cytoskeleton stability, as well as the regulation of cell division, differentiation, and apoptosis [98]. In the current study, a coexpressed network analyzed by WGCNA indicated that 9 GhSK genes were co-expressed with one or more other G. hirsutum genes. Additionally, a KEGG enrichment analysis $(p<0.05)$ revealed that these 9 GhSK genes could be involved in stress responses, phytohormone signal transductions, and/or the Wnt signaling pathway. Moreover, the genes co-expressed in the brown module containing GhSK32 and GhSK34 were significantly enriched for protein phosphorylation (GO:0006468), protein kinase activity (GO:0004672), integral component of membrane (GO:0016021), Calcium ion binding (GO:0005509), transmembrane transport (GO:0055085) and enzyme inhibitor activity (GO:0004857). The genes co-expressed with GhSK41 and GhSK42 were enriched for functions related to cytoskeleton organization (GO:0007010) and microtubule binding (GO:0008017). Cortical microtubules and newly deposited cellulose microfibrils are transversely oriented relative to the growth axis of fiber cell, and are critical for longitudinal expansion [99]. In addition, the temporal and spatial $\mathrm{Ca}^{2+}$ concentration changes in the fiber tip are converted through calcium sensors, such as calmodulins, calcium-dependent protein kinases, and calcineurin B-like proteins that have been shown to be necessary for maintaining fast cell elongation [23]. Furthermore, ethylene has also been shown to promote fiber elongation by activating genes involved in cytoskeleton organization and cell wall loosening $[23,90]$. These findings may help to clarify the potential functions of GhSKs in fiber development and will lay the foundations for further molecular and functional analysis of GSK genes in cotton.

\section{Conclusions}

Previous research has shown that GSK genes have significant functions in regulating plant growth and development, as well as responses to various abiotic stresses. In the present study, GSK genes were shown to be highly conserved among Arabidopsis, rice and three cotton species (G. hirsutum, G. raimondii, and G. arboreum). Whole genome duplication was speculated as the major impetus for the expansion of the GSK gene family in cotton. Additionally, the duplicated GhSK genes might have experienced functional diversification as the duplicated gene pairs exhibited disparate expression patterns in different tissues and organs. Further, some GSK gene members were shown to be involved in fiber development as well as stress responses.

\section{Additional files}

Additional file 1: Table S1.1 Gene accession numbers and sample information of transcriptome data used in our research. Table S1.2 FPKM values of transcriptome data used in our research. (XLSX 16765 kb)

Additional file 2: Table S2. FPKM values of different tissues obtained from published transcriptome data. (XLSX $16 \mathrm{~kb}$ )

Additional file 3: Figure S1. Multiple sequence alignments of the kinase domains of Arabidopsis, rice, and cotton. Amino acids are shaded in black, dark and light grey based on sequence similarity (high to low). The kinase-inactivating mutation bin2 that suppresses gain of function are highlighted in light green. The conserved phosphate-binding residues are highlighted in orange. The conversed tyrosine phosphorylated residue is highlighted in red. The conserved TRRE box in which the three residues (T, E, and E) that lead to BIN2 gain of function are included are highlighted in green. (PDF $3258 \mathrm{~kb}$ )

Additional file 4: Figure S2. Phylogenetic analysis of GSK3 proteins in Arabidopsis, rice, and species of cotton. 10 AtSKs, 15 OsSKs, 20 GhSKs, 10 GaSKs, and $10 \mathrm{GrSKs}$ are divided into four clades. The four clades are respectively colored in cyan, reddish brown, violet, and green. The tree was constructed by Minimum-Evolution using Poisson model of MEGA 6.0. (PDF $301 \mathrm{~kb}$ )

Additional file 5: Figure S3. Motif logos of 16 conserved motifs found in cotton GSK proteins. (PDF $817 \mathrm{~kb}$ )

Additional file 6: Table S3. Orthologous GSK genes identified in G. hirsutum, G. arboreum and G. raimondii. Orthologous genes were highlighted on condition that they were coincident with the results analyzed by MCScanX software: orthologous gene pairs between $G$. hirsutum and G. arboreum were highlighted in red, G. hirsutum and G. raimondii in blue, as well as G. arboreum and G. raimondii in orange. (XLSX $18 \mathrm{~kb})$ 
Additional file 7: Figure S4. Cis-regulatory elements predicted in the promoter regions of cotton GSK genes. (PDF $173 \mathrm{~kb}$ )

Additional file 8: Table S4. Cis-regulatory elements found in the promoter regions of cotton GSK genes. (XLSX $33 \mathrm{~kb}$ )

Additional file 9: Figure S5. The GO (a) and KEGG (b) enrichment of weighted co-expressed genes of the brown module (GhSK32) as predicted by "WGCNA" R package with published transcriptomics data. (PDF $149 \mathrm{~kb}$ )

Additional file 10: Figure S6. The weighted gene co-expression analysis sub-network of GhSK32 and GhSK34 were visualized by Cytoscape software. (PDF $7454 \mathrm{~kb}$ )

Additional file 11: Table S5.1 GO annotations of the co-expressed genes involved in the weighted gene co-expression sub-network of GhSKs. Table S5.2 KEGG annotations of the co-expressed genes involved in the weighted gene co-expression sub-network of GhSKs. Table S5.3 Linkers co-expressed with GhSK32 and GhSK34. (XLSX 201 kb)

Additional file 12: Figure S7. 20 GhSKs expression patterns in response to $B L$ treatment were analyzed by qRT-PCR. The relative expression levels of $\mathrm{CK}(0 \mathrm{~h})$ were normalized to 1 . Data are the mean $\pm \mathrm{SE}$ of three independent experiments. (PDF $459 \mathrm{~kb})$

\section{Abbreviations}

A. thaliana: Arabidopsis thaliana; ABA: Abscisic acid; BIN2: BrassinosteroidInsensitive 2; BL: Brassinolide; BLAT: BLAST-Like Alignment Tool; BR: Brassinosteroid; Dpa: Day post-anthesis; G6PD: Glc-6-phosphate dehydrogenase; GA: Gibberellins; GaSKs: Gossypium arboreum Shaggy/GSK3like kinases; GhSKs: Gossypium hirsutum Shaggy/GSK3-like kinases; GO: Gene Ontology; GrSKs: Gossypium raimondii Shaggy/GSK3-like kinases; GSK3: Glycogen synthase 3/shaggy-like kinase; KEGG: Kyoto Encyclopedia of Genes and Genomes; PEG: Polyethylene glycol; qRT-PCR: Quantitative realtime polymerase chain reaction; WGCNA: Weighted gene co-expression network analysis

\section{Acknowledgments}

The authors would like to thank the previous researchers and communities (particularly Tianzhen Zhang et al., Nanjing Agricultural University) to submit the transcriptome data of the upland cotton TM-1 to NCBI SRA databases. Special thanks to Dr. Xiaoyang Ge for his insightful comments and suggestions that helped us to improve our manuscript.

\section{Funding}

This work was supported by the National Natural Science Foundation of China (No. 31501345) in writing the manuscript and Young Elite Scientist Sponsorship Program by CAST supported the funds for the research but was not involved in data collection and analysis, or the preparation or publication of the manuscript.

\section{Availability of data and materials}

The data generated or analyzed in this study are included in this article and its Additional materials. And the Genome sequence and annotation datasets that supported the findings of this study are available in: A. thaliana: http://www.arabidopsis.org.

O. sativa: http://plants.ensembl.org/index.html.

G. hirsutum: https://www.cottongen.org

G. arboreum: PacBio-Gar-Assembly-v1.0, ftp://bioinfo.ayit.edu.cn/downloads/

G. raimondii: https://www.cottongen.org

\section{Authors' contributions}

FGL and ZRY designed the experiments. LLW performed the data analysis and drafted the manuscript. ZEY provided and analyzed the transcriptome data. BZ, DQY, and QG conducted the abiotic stress experiments and provided the organs/tissues of CRI24. GQ and YJL contributed technical support in writing and editing the manuscript. LLL, YL, and JL prepared the cotton seedlings. All of the authors read and approved the final manuscript.

\section{Ethics approval and consent to participate}

The plant materials (including seeds) were collected from Zhengzhou Research base, State key Laboratory of Cotton Biology, Institute of Cotton Research, CAAS. The experimental research on plants, including collection of plant material, was complied with the institutional, national, or international guidelines.

\section{Consent for publication}

Not applicable.

\section{Competing interests}

The authors declare that they have no competing interests.

\section{Publisher's Note}

Springer Nature remains neutral with regard to jurisdictional claims in published maps and institutional affiliations.

\section{Author details}

${ }^{1}$ State Key Laboratory of Cotton Biology, Key Laboratory of Biological and Genetic Breeding of Cotton, Institute of Cotton Research, Chinese Academy of Agricultural Sciences, Anyang 455000, Henan, China. ${ }^{2}$ National Key Laboratory of Crop Genetic Improvement and National Centre of Plant Gene Research, Huazhong Agricultural University, Wuhan 430070, People's Republic of China.

Received: 6 July 2018 Accepted: 14 November 2018 Published online: 04 December 2018

\section{References}

1. Yoo MJ, Albert VA, Soltis PS, Soltis DE. Phylogenetic diversification of glycogen synthase kinase 3/SHAGGY-like kinase genes in plants. BMC Plant Biol. 2006;6:3.

2. Saidi Y, Hearn TJ, Coates JC. Function and evolution of 'green' GSK3/ Shaggy-like kinases. Trends Plant Sci. 2012;17(1):39-46.

3. Orena SJ, Torchia AJ, Garofalo RS. Inhibition of glycogen-synthase kinase 3 stimulates glycogen synthase and glucose transport by distinct mechanisms in 3T3-L1 adipocytes. J Bio Chem. 2000;275(21):15765-72.

4. Rylatt DB, Aitken A, Bilham T, Condon GD, Embi N, Cohen P. Glycogen synthase from rabbit skeletal muscle. Amino acid sequence at the sites phosphorylated by glycogen synthase kinase-3, and extension of the $\mathrm{N}$ terminal sequence containing the site phosphorylated by phosphorylase kinase. Eur J Biochem. 1980;107(2):529-37.

5. Simpson $P, M E M L, J M d P$, Ripoll P. Stripes of positional homologies across the wing blade of Drosophila melanogaster. Development. 1988; 103:391-401.

6. Yang XG, Liang WH, Li F, Ma WS. OsGSK3 is a novel GSK3/SHAGGY-like gene from Oryza sativa L., involved in abiotic stress signaling. Pak J Bot. 2012; 44(5):1491-6.

7. Petersen $C P$, Reddien PW. Wnt signaling and the polarity of the primary body axis. Cell. 2009;139(6):1056-68.

8. He X, Saint-Jeannet JP, Woodgett JR, Varmus HE, Dawid IB. Glycogen synthase kinase-3 and dorsoventral patterning in Xenopus embryos. Nature. 1995;374(6523):617-22

9. Wu D, Pan W. GSK3: a multifaceted kinase in Wnt signaling. Trends Biochem Sci. 2010;35(3):161-8.

10. Woodgett JR, Cohen P. Multisite phosphorylation of glycogen synthase. Molecular basis for the substrate specificity of glycogen synthase kinase-3 and casein kinase-II (glycogen synthase kinase-5). Biochimica Biophys Acta. 1984;788(3):339-47.

11. Dornelas MC, van Lammeren AA, Kreis M. Arabidopsis thaliana SHAGGYrelated protein kinases (AtSK11 and AtSK12) function in perianth and gynoecium development. Plant J. 2000;21(5):419-29.

12. Charrier B, Champion A, Henry $Y$, Kreis M. Expression profiling of the whole Arabidopsis shaggy-like kinase multigene family by real-time reverse transcriptase-polymerase chain reaction. Plant Physiol. 2002;130(2):577-90.

13. Tavares R, Vidal J, van Lammeren A, Kreis M. AtSKE, a plant homologue of SGG_GSK-3 marks developing tissues in Arabidopsis thaliana. Plant Mol Biol. 2002;50:261-71.

14. Claisse G, Charrier B, Kreis M. The Arabidopsis thaliana GSK3/Shaggy like kinase AtSK3-2 modulates floral cell expansion. Plant Mol Biol. 2007;64(1-2): $113-24$.

15. Dal Santo S, Stampfl H, Krasensky J, Kempa S, Gibon Y, Petutschnig E, et al. Stress-induced GSK3 regulates the redox stress response by phosphorylating glucose-6-phosphate dehydrogenase in Arabidopsis. Plant Cell. 2012;24(8):3380-92. 
16. Li J, Nam KH. Regulation of Brassinosteroid Signaling by a GSK3/SHAGGYLike Kianse. Science. 2002;295:1299-301.

17. Wendel JF, Cronn RC. Polyploidy and the evolutionary history of cotton. Adv Agron. 2003;78:139-86.

18. Tiwari SC, Wilkins TA. Cotton (Gossypium hirsutum) seed trichomes expand via diffuse growing mechanism. Can J Bot. 1995;73(5):746-57.

19. Paterson $A H$, Wendel JF, Gundlach $H$, Guo H, Jenkins J, Jin $D$, et al. Repeated polyploidization of Gossypium genomes and the evolution of spinnable cotton fibres. Nature. 2012;492(7429):423-7.

20. Yang SS, Cheung F, Lee JJ, Ha M, Wei NE, Sze S-H, et al. Accumulation of genome-specific transcripts, transcription factors and phytohormonal regulators during early stages of fiber cell development in allotetraploid cotton. Plant J. 2006;47(5):761-75.

21. Sun Y, Veerabomma S, Abdel-Mageed HA, Fokar M, Asami T, Yoshida S, Allen RD. Brassinosteroid regulates fiber development on cultured cotton ovules. Plant Cell Physiol. 2005;46(8):1384-91.

22. Tang W, Tu L, Yang X, Tan J, Deng F, Hao J, Guo K, Lindsey K, Zhang X. The calcium sensor GhCaM7 promotes cotton fiber elongation by modulating reactive oxygen species (ROS) production. New Phytol. 2014;202(2):509-20.

23. Qin YM, Zhu YX. How cotton fibers elongate: a tale of linear cell-growth mode. Curr Opin Plant Biol. 2011:14(1):106-11.

24. Yang Z, Zhang C, Yang X, Liu K, Wu Z, Zhang X, et al. PAG1, a cotton brassinosteroid catabolism gene, modulates fiber elongation. New Phytol. 2014;203(2):437-48.

25. Seagull RW, Giavalis S. Pre- and Post-Anthesis Application of Exogenous Hormones Alters Finer Production in Gossypium hirsutum L. Cultivar Maxxa GTO. J Cotton Sci. 2004;8:105-11.

26. Nemhauser JL, Hong F, Chory J. Different plant hormones regulate similar processes through largely nonoverlapping transcriptional responses. Cell. 2006;126(3):467-75.

27. Zhang S, Cai Z, Wang X. The primary signaling outputs of brassinosteroids are regulated by abscisic acid signaling. Proc Natl Acad Sci U S A. 2009; 106(11):4543-8.

28. Chaiwanon J, Wang ZY. Spatiotemporal brassinosteroid signaling and antagonism with auxin pattern stem cell dynamics in Arabidopsis roots. Curr Biol. 2015;25(8):1031-42.

29. Liu J, Rowe J, Lindsey K. Hormonal crosstalk for root development: a combined experimental and modeling perspective. Front Plant Sci. 2014;5:116.

30. Vandenbussche F, Callebert P, Zadnikova P, Benkova E, Van Der Straeten D. Brassinosteroid control of shoot gravitropism interacts with ethylene and depends on auxin signaling components. Am J Bot. 2013;100(1):215-25.

31. Sun Y, Fan XY, Cao DM, Tang W, He K, Zhu JY, et al. Integration of brassinosteroid signal transduction with the transcription network for plant growth regulation in Arabidopsis. Dev Cell. 2010;19(5):765-77.

32. Aleman L, Kitamura J, Abdel-mageed H, Lee J, Sun Y, Nakajima M, Ueguchi-Tanaka M, Matsuoka M, Allen RD. Functional analysis of cotton orthologs of GA signal transduction factors GID1 and SLR1. Plant Mol Biol. 2008;68(1-2):1-16.

33. Li F, Fan G, Lu C, Xiao G, Zou C, Kohel RJ, et al. Genome sequence of cultivated Upland cotton (Gossypium hirsutum TM-1) provides insights into genome evolution. Nat Biotechnol. 2015;33(5):524-30.

34. Zhang T, Hu Y, Jiang W, Fang L, Guan X, Chen J, et al. Sequencing of allotetraploid cotton (Gossypium hirsutum L. acc. TM-1) provides a resource for fiber improvement. Nat Biotechnol. 2015;33(5):531-7.

35. Wang $K$, Wang Z, Li F, Ye W, Wang J, Song G, et al. The draft genome of a diploid cotton Gossypium raimondii. Nat Genet. 2012;44(10):1098-103.

36. Li F, Fan G, Wang K, Sun F, Yuan Y, Song G, et al. Genome sequence of the cultivated cotton Gossypium arboreum. Nat Genet. 2014;46(6):567-72.

37. Lee JJ, Woodward AW, Chen ZJ. Gene expression changes and early events in cotton fibre development. Ann Bot. 2007;100(7):1391-401.

38. Zhang B, Liu J, Yang ZE, Chen EY, Zhang CJ, Zhang XY, Li FG. Genome-wide analysis of GRAS transcription factor gene family in Gossypium hirsutum L. BMC Genomics. 2018;19(1):348.

39. Yang Z, Gong Q, Qin W, Yang Z, Cheng Y, Lu L, et al. Genome-wide analysis of WOX genes in upland cotton and their expression pattern under different stresses. BMC Plant Biol. 2017:17(1):113.

40. Livak KJ, Schmittgen TD. Analysis of relative gene expression data using real-time quantitative PCR and the 2(-Delta Delta C(T)) Method. Methods. 2001;25(4):402-8.

41. Zerbino DR, Achuthan P, Akanni W, Amode MR, Barrell D, Bhai J, et al. Ensembl 2018. Nucleic Acids Res. 2018;46(D1):D754-61.
42. Yu J, Jung S, Cheng CH, Ficklin SP, Lee T, Zheng P, Jones D, Percy RG, Main D. CottonGen: a genomics, genetics and breeding database for cotton research. Nucleic Acids Res. 2014;42(Database issue):D1229-36.

43. Du X, Huang G, He S, Yang Z, Sun G, Ma X, et al. Resequencing of 243 diploid cotton accessions based on an updated $\mathrm{A}$ genome identifies the genetic basis of key agronomic traits. Nat Genet. 2018;50(6):796-802.

44. Finn RD, Coggill P, Eberhardt RY, Eddy SR, Mistry J, Mitchell AL, et al. The Pfam protein families database: towards a more sustainable future. Nucleic Acids Res. 2016:44(D1):D279-85.

45. Youn JH, Kim TW. Functional insights of plant GSK3-like kinases: multitaskers in diverse cellular signal transduction pathways. Mol Plant. 2015;8(4): $552-65$.

46. Kumar S, Stecher G, Peterson D, Tamura K. MEGA-CC: computing core of molecular evolutionary genetics analysis program for automated and iterative data analysis. Bioinformatics. 2012;28(20):2685-6.

47. Wang S, Chen J, Zhang W, Hu Y, Chang L, Fang L, et al. Sequence-based ultra-dense genetic and physical maps reveal structural variations of allopolyploid cotton genomes. Genome Biol. 2015;16(1):108.

48. Bailey TL, Elkan C. The value of prior knowledge in discovering motifs with MEME. Proc Int Conf Intell Syst Mol Biol. 1995;3:21-9.

49. Doerks T, Copley RR, Schultz J, Ponting CP, Bork P. Systematic identification of novel protein domain families associated with nuclear functions. Genome Res. 2002;12(1):47-56.

50. Krzywinski M, Schein J, Birol I, Connors J, Gascoyne R, Horsman D, Jones SJ, Marra MA. Circos: an information aesthetic for comparative genomics. Genome Res. 2009;19(9):1639-45.

51. Lescot M, Dehais P, Thijs G, Marchal K, Moreau Y, Van de Peer $Y$, Rouze $P$, Rombauts S. PlantCARE, a database of plant cis-acting regulatory elements and a portal to tools for in silico analysis of promoter sequences. Nucleic Acids Res. 2002:30(1):325-7.

52. Gasteiger E, Hoogland C, Gattiker A, Duvaud S, Wilkins MR, Appel RD, Bairoch A. Protein Identification and Analysis Tools on the ExPASy Server. In: The Proteomics Protocols Handbook; 2005. p. 571-607.

53. Briesemeister S, Rahnenfuhrer J, Kohlbacher O. YLoc--an interpretable web server for predicting subcellular localization. Nucleic Acids Res. 2010;38(Web Server issue):W497-502.

54. Wang M, Yuan D, Tu L, Gao W, He Y, Hu H, et al. Long noncoding RNAs and their proposed functions in fibre development of cotton (Gossypium spp.). New Phytol. 2015;207(4):1181-97.

55. Langfelder P, Horvath S. WGCNA: an R package for weighted correlation network analysis. BMC Bioinformatics. 2008;9:559.

56. Shannon P, Markiel A, Ozier O, Baliga NS, Wang JT, Ramage D, Amin N, Schwikowski B, Ideker T. Cytoscape: a software environment for integrated models of biomolecular interaction networks. Genome Res. 2003;13(11): 2498-504.

57. Le DT, Nishiyama R, Watanabe Y, Mochida K, Yamaguchi-Shinozaki K, Shinozaki K, Tran LS. Genome-wide survey and expression analysis of the plant-specific NAC transcription factor family in soybean during development and dehydration stress. DNA Res. 2011;18(4):263-76.

58. Dornelas MC, Wittich P, von Recklinghausen I, van Lammeren A, Kreis M. Characterization of three novel members of the Arabidopsis SHAGGY-related protein kinase (ASK) multigene family. Plant Mol Biol. 1999:39(1):137-47.

59. Jonak C, Hirt H. Glycogen synthase kinase 3/SHAGGY-like kinases in plants: an emerging family with novel functions. Trends Plant Sci. 2002; 7(10):457-61.

60. Cannon SB, Mitra A, Baumgarten A, Young ND, May G. The roles of segmental and tandem gene duplication in the evolution of large gene families in Arabidopsis thaliana. BMC Plant Biol. 2004:4:10.

61. Qiao L, Zhang X, Han X, Zhang L, Li X, Zhan H, et al. A genome-wide analysis of the auxin/indole-3-acetic acid gene family in hexaploid bread wheat (Triticum aestivum L.). Front Plant Sci. 2015;6:770.

62. Yue Z, Li H-T, Yang Y, Hussain S, Zheng C-H, Xia J, Chen Y. OncotargetIdentification of breast cancer candidate genes using gene co-expression and protein-protein interaction information. Oncotarget. 2016;7:36092-100.

63. Aoki K, Ogata Y, Shibata D. Approaches for extracting practical information from gene co-expression networks in plant biology. Plant Cell Physiol. 2007; 48(3):381-90.

64. Hanks SK, Quinn AM, Hunter T. The protein kinase family: conserved features and deduced phylogeny of the catalytic domains. Science. 1988; 241(4861):42-52. 
65. Venkatesh J, Jahn M, Kang BC. Genome-Wide Analysis and Evolution of the Pto-Like Protein Kinase (PLPK) Gene Family in Pepper. PloS One. 2016;11(8): e0161545.

66. Cao J, Jiang M, Li P, Chu Z. Genome-wide identification and evolutionary analyses of the PP2C gene family with their expression profiling in response to multiple stresses in Brachypodium distachyon. BMC Genomics. 2016;17(1):175.

67. Dornelas MC, Lejeune B, Dron M, Kreis M. The Arabidopsis SHAGGY-related protein kinase (ASK) gene family: structure, organization and evolution. Gene. 1998;212(2):249-57.

68. Wang M, Yue H, Feng K, Deng P, Song W, Nie X. Genome-wide identification, phylogeny and expressional profiles of mitogen activated protein kinase kinase kinase (MAPKKK) gene family in bread wheat (Triticum aestivum L.). BMC Genomics. 2016:17:668.

69. Hur EM, Zhou FQ. GSK3 signaling in neural development. Nat Rev Neurosci. 2010;11(8):539-51.

70. Double BW, Woodgeet JR. GSK-3: trick of the trade for multi-tasking kinase J Cell Sci. 2003:116:1175-86.

71. Kohel RJ. Genetic nomenclature in cotton. J Hered. 1973;64(5):291.

72. Forde JE, Dale TC. Glycogen synthase kinase 3: a key regulator of cellular fate. Cell Mol Life Sci. 2007;64(15):1930-44.

73. Jiang M, Wen F, Cao JM, Li P, She J, Chu ZQ. Genome-wide exploration of the molecular evolution and regulatory network of mitogen-activated protein kinase cascades upon multiple stresses in Brachypodium distachyon. BMC Genomics. 2015;16(1):228.

74. Lochhead PA, Kinstrie R, Sibbet G, Rawjee T, Morrice N, Cleghon V. A chaperonedependent GSK3beta transitional intermediate mediates activation-loop autophosphorylation. Mol Cell. 2006;24(4):627-33.

75. Thomas GM, Frame S, Goedert M, Nathke I, Polakis P. A GSK3-binding peptide from FRAT1 selectively inhibits the GSK3-catalysed phosphorylation of Axin and L-catenin. FEBS Letters. 1999;458:247-51.

76. Tatusov RL, Koonin EV, Lipman DJ. A genomic perspective on protein families. Science. 1997;278(5338):631-7.

77. Heidelberg JF, Paulsen IT, Nelson KE, Gaidos EJ, Nelson WC, Read TD, et al. Genome sequence of the dissimilatory metal ion-reducing bacterium Shewanella oneidensis. Nat Biotechnol. 2002;20(11):1118-23.

78. Zhang JZ. Evolution by gene duplication: an update. Trens Ecol Evol. 2003; 18(6):292-8

79. Achaz G, Coissac E, Viari A, Netter P. Analysis of intrachromosomal duplications in yeast Saccharomyces cerevisiae: a possible model for their origin. Mol Biol Evol. 2000;17(8):1268-75.

80. Altenhoff AM, Dessimoz C. Phylogenetic and functional assessment of orthologs inference projects and methods. PLoS Comput Biol. 2009;5(1): e1000262.

81. Perez-Perez JM, Ponce MR, Micol JL. The UCU1 Arabidopsis gene encodes a SHAGGY/GSK3-like kinase required for cell expansion along the proximodistal axis. Dev Biol. 2002;242(2):161-73.

82. Kim TW, Guan S, Sun Y, Deng Z, Tang W, Shang JX, Sun Y, Burlingame AL, Wang ZY. Brassinosteroid signal transduction from cell-surface receptor kinases to nuclear transcription factors. Nat Cell Biol. 2009;11(10):1254-60.

83. Saini S, Sharma I, Pati PK. Versatile roles of brassinosteroid in plants in the context of its homeostasis, signaling and crosstalks. Front Plant Sci. 2015;6:950.

84. Vert G, Nemhauser JL, Geldner N, Hong F, Chory J. Molecular mechanisms of steroid hormone signaling in plants. Annu Rev Cell Dev Biol. 2005;21: 177-201.

85. Belkhadir Y, Wang X, Chory J. Arabidopsis Brassinosteroid Signaling Pathway. Sci STKE. 2006;2006(364):cm5.

86. Oh E, Zhu JY, Bai MY, Arenhart RA, Sun Y, Wang ZY. Cell elongation is regulated through a central circuit of interacting transcription factors in the Arabidopsis hypocotyl. Elife. 2014;3:e03031.

87. Cho H, Ryu H, Rho S, Hill K, Smith S, Audenaert D, et al. A secreted peptide acts on BIN2-mediated phosphorylation of ARFs to potentiate auxin response during lateral root development. Nat Cell Biol. 2014;16(1):66-76.

88. Singh $B$, Cheek HD, Haigler $\mathrm{CH}$. A synthetic auxin (NAA) suppresses secondary wall cellulose synthesis and enhances elongation in cultured cotton fiber. Plant Cell Rep. 2009;28(7):1023-32.

89. Zhang M, Zheng X, Song S, Zeng Q, Hou L, Li D, et al. Spatiotemporal manipulation of auxin biosynthesis in cotton ovule epidermal cells enhances fiber yield and quality. Nat Biotechnol. 2011;29(5):453-8.

90. Shi YH, Zhu SW, Mao XZ, Feng JX, Qin YM, Zhang L, et al. Transcriptome profiling, molecular biological, and physiological studies reveal a major role for ethylene in cotton fiber cell elongation. Plant Cell. 2006;18(3):651-64.
91. Koh S, Lee SC, Kim MK, Koh JH, Lee S, An G, Choe S, Kim SR. T-DNA tagged knockout mutation of rice OsGSK1, an orthologue of Arabidopsis BIN2, with enhanced tolerance to various abiotic stresses. Plant Mol Biol. 2007;65(4): 453-66.

92. Piao HL, Lim JH, Kim SJ, Cheong GW, Hwang I. Constitutive over-expression of AtGSK1 induces $\mathrm{NaCl}$ stress responses in the absence of $\mathrm{NaCl}$ stress and results in enhanced $\mathrm{NaCl}$ tolerance in Arabidopsis. Plant J. 2001;27(4):305-14.

93. Richard O, Paquet N, Haudecoeur E, Charrier B. Organization and expression of the GSK3/shaggy kinase gene family in the moss Physcomitrella patens suggest early gene multiplication in land plants and an ancestral response to osmotic stress. J Mol Evol. 2005:61(1):99-113.

94. Kempa S, Rozhon W, Samaj J, Erban A, Baluska F, Becker T, et al. A plastidlocalized glycogen synthase kinase 3 modulates stress tolerance and carbohydrate metabolism. Plant J. 2007;49(6):1076-90.

95. Patade VY, Rai AN, Suprasanna P. Expression analysis of sugarcane shaggylike kinase (SuSK) gene identified through CDNA subtractive hybridization in sugarcane (Saccharum officinarum L.). Protoplasma. 2011;248(3):613-21.

96. Chen GP, Ma WS, Huang ZJ, Xu T, Xue YB, Shen YZ. Isolation and characterization of TaGSK1 involved in wheat salt tolerance. Plant Sci. 2003; 165(6):1369-75

97. Christov NK, Christova PK, Kato H, Liu Y, Sasaki K, Imai R. TaSK5, an abiotic stress-inducible GSK3/shaggy-like kinase from wheat, confers salt and drought tolerance in transgenic Arabidopsis. Plant Physiol Biochem. 2014;84 251-60.

98. Frame S, Cohen P. GSK3 takes centre stage more than 20 years after its discovery. Biochem J. 2001;359:1-16.

99. Seagull RW. The effects of microtubule and microfilament disrupting agents on cytoskeletal arrays and wall deposition in developing cotton fibers. Protoplasma. 1990;159:44-59.

\section{Ready to submit your research? Choose BMC and benefit from:}

- fast, convenient online submission

- thorough peer review by experienced researchers in your field

- rapid publication on acceptance

- support for research data, including large and complex data types

- gold Open Access which fosters wider collaboration and increased citations

- maximum visibility for your research: over $100 \mathrm{M}$ website views per year

At $\mathrm{BMC}$, research is always in progress.

Learn more biomedcentral.com/submissions 